\title{
Inverse stellar population age gradients of post-starburst galaxies at $z=0.8$ with LEGA-C
}

Francesco D'Eugenio ${ }^{1 \star}$, Arjen van der $\mathrm{Wel}^{1,2}$, Po-Feng Wu (吴柏鋒) ${ }^{3,2}$, Tania M. Barone $^{4,5,6}$, Josha van Houdt ${ }^{2}$, Rachel Bezanson ${ }^{7}$, Caroline M. S. Straatman ${ }^{1}$, Camilla Pacifici ${ }^{8}$, Adam Muzzin ${ }^{9}$, Anna Gallazzi ${ }^{10}$, Vivienne Wild ${ }^{11}$, David Sobral $^{12}$, Eric F. Bell ${ }^{13}$, Stefano Zibetti ${ }^{10}$ Lamiya Mowla ${ }^{14}$ and Marijn Franx ${ }^{15}$

\author{
${ }^{1}$ Sterrenkundig Observatorium, Universiteit Gent, Krijgslaan 281 S9, B-9000 Gent, Belgium \\ ${ }^{2}$ Max-Planck-Institut für Astronomie, Königstuhl 17, D-69117, Heidelberg, Germany \\ ${ }^{3}$ National Astronomical Observatory of Japan, 2-21-1 Osawa, Mitaka, Tokyo 181-8588, Japan \\ ${ }^{4}$ Research School of Astronomy and Astrophysics, Australian National University, Canberra, ACT 2611, Australia \\ ${ }^{5}$ Sydney Institute for Astronomy, School of Physics, The University of Sydney, NSW, 2006, Australia \\ ${ }^{6}$ ARC Centre of Excellence for All Sky Astrophysics in 3 Dimensions (ASTRO 3D), Australia \\ ${ }^{7}$ Department of Physics and Astronomy and PITT PACC, University of Pittsburgh, Pittsburgh, PA 15260, USA \\ ${ }^{8}$ Space Telescope Science Institute, 3700 San Martin Drive, Baltimore, MD 21218, USA \\ ${ }^{9}$ Department of Physics and Astronomy, York University, 4700 Keele St., Toronto, Ontario, M3J 1P3, Canada \\ ${ }^{10}$ INAF-Osservatorio Astrofisico di Arcetri, Largo Enrico Fermi 5, I-50125 Firenze, Italy \\ ${ }^{11}$ School of Physics and Astronomy, University of St Andrews, North Haugh, St Andrews KY16 9SS, UK (SUPA) \\ ${ }^{12}$ Department of Physics, Lancaster University, Lancaster LA1 $4 Y B$, UK \\ ${ }^{13}$ Department of Astronomy, University of Michigan, Ann Arbor, MI 48109, USA \\ ${ }^{14}$ Astronomy Department, Yale University, New Haven, CT 06511, US \\ ${ }^{15}$ Leiden Observatory, P.O. Box 9513, 2300 RA, Leiden, The Netherlands
}

Accepted 2020 Jun 29. Received 2020 June 23; in original form 2020 March 25

\begin{abstract}
We use deep, spatially resolved spectroscopy from the LEGA-C Survey to study radial variations in the stellar population of 17 spectroscopically-selected post-starburst (PSB) galaxies. We use spectral fitting to measure two Lick indices, $\mathrm{H} \delta_{\mathrm{A}}$ and Fe4383, and find that, on average, PSB galaxies have radially decreasing $\mathrm{H} \delta_{\mathrm{A}}$ and increasing Fe4383 profiles. In contrast, a control sample of quiescent, non-PSB galaxies in the same mass range shows outwardly increasing $\mathrm{H} \delta_{\mathrm{A}}$ and decreasing Fe4383. The observed gradients are weak $\left(\approx-0.2 \AA / R_{e}\right)$, mainly due to seeing convolution. A two-SSP model suggests intrinsic gradients are as strong as observed in local PSB galaxies $\left(\approx-0.8 \AA / R_{e}\right)$. We interpret these results in terms of inside-out growth (for the bulk of the quiescent population) vs star formation occurring last in the centre (for PSB galaxies). At $z \approx 0.8$, central starbursts are often the result of gas-rich mergers, as evidenced by the high fraction of PSB galaxies with disturbed morphologies and tidal features (40\%). Our results provide additional evidence for multiple paths to quiescence: a standard path, associated with inside-out disc formation and with gradually decreasing star-formation activity, without fundamental structural transformation, and a fast path, associated with centrally-concentrated starbursts, leaving an inverse age gradient and smaller half-light radius.
\end{abstract}

Key words: galaxies: formation, galaxies: evolution, galaxies: starburst, galaxies: high redshift, galaxies: fundamental parameters, galaxies: structure

\section{INTRODUCTION}

* E-mail: francesco.deugenio@gmail.com

At any given time, star-forming (SF) galaxies form a sequence in the mass-size plane (Shen et al. 2003, 
van der Wel et al. 2014); this mass-size relation is such that, at fixed stellar mass, SF galaxies are systematically larger than non-star-forming galaxies (hereafter: quiescent, or $\mathrm{Q}$ galaxies). Moreover, the average size of both SF and Q galaxies increases with cosmic time (van der Wel et al. 2009; Fagioli et al. 2016; Williams et al. 2017). Given these properties, it is reasonable to assume that galaxies that have recently become quiescent have approximately the same size as SF galaxies of the same mass. This expectation is indeed consistent with the finding that, at fixed stellar mass, the youngest $\mathrm{Q}$ galaxies are also the largest (Wu et al. 2018).

There is however a class of objects, called post-starburst (PSB) galaxies, that have recently become quiescent, yet contrary to the above expectations are both: (i) smaller than coeval Q galaxies, and (ii) much smaller than coeval SF galaxies (e.g. Whitaker et al. 2012, Almaini et al. 2017, Wu et al. 2018). Observationally, PSB galaxies (Dressler \& Gunn 1983; Couch \& Sharples 1987) present strong Balmerline absorption (typical of young, $0.3-1$ Gyr-old stars) but lack $\mathrm{H} \alpha$ emission (which excludes recent, $\lesssim 10$ Myr star formation). Together, these two properties suggest that PSB galaxies stopped forming stars both rapidly (faster than $\approx 1 \mathrm{Gyr}$ ) and recently (within $\approx 1 \mathrm{Gyr}$ of their look-back time). The empirical conjunction of compact structure with a rapid and recent transition to quiescence, suggests that PSB galaxies followed a special evolutionary path, either an extreme version of normal galaxy evolution, or some entirely different channel.

In the local Universe, PSB galaxies are empirically associated either with galaxy mergers (e.g. Zabludoff et al. 1996; Bekki et al. 2001; Yang et al. 2004; Goto 2005; Yang et al. 2008; Pracy et al. 2009; Wild et al. 2009; Pawlik et al. 2018) or with ram-pressure stripping in dense environments (Dressler et al. 1999; Poggianti et al. 1999; Tran et al. 2004; Poggianti et al. 2009; Paccagnella et al. 2019). They show a range of kinematic properties: from dispersion-dominated kinematics reminiscent of quiescent galaxies (and consistent with the outcome of mergers; Hiner \& Canalizo 2015) to rotation-supported systems (e.g. Norton et al. 2001; Pracy et al. 2013; Owers et al. 2019). Chen et al. (2019) find that stellar kinematics depend on the location of the PSB regions within the target galaxy. In any case, even accounting for their relatively short visibility time, local PSB galaxies represent a marginal mode of galaxy evolution (Rowlands et al. 2018).

However, in the high-redshift Universe, PSB galaxies could be different. Firstly, it appears that the fraction of PSB galaxies increases with cosmic time (e.g. Dressler et al. 1999, Poggianti et al. 1999, Wild et al. 2016, but see e.g. Balogh et al. 1997, Balogh et al. 1999, Muzzin et al. 2012 for a different view). Even if the fraction of PSB galaxies stayed constant, dense environments become rarer with increasing redshift (e.g. Carlberg et al. 1997; Younger et al. 2005), hence the physics underlying low- and high-redshift PSB galaxies could be different. This is not a surprising possibility, because the definition of PSB galaxies is purely empirical, and different quenching mechanisms could ostensibly leave similar or identical signatures. In addition, there is evidence for different structural properties between $z<1$ and $z>1$ PSB galaxies (Maltby et al. 2018), suggesting that redshift evolution might involve different physical processes.

A possible explanation of the observed properties of
PSB galaxies is a central starburst in a previously normal galaxy. A significant amount of star formation inside $\approx 1 \mathrm{kpc}$ from the centre of a galaxy can reduce its previous half-light radius, thus explaining the small observed size of PSB galaxies (Wu et al. 2020). At the same time, central starbursts are likely to undergo rapid quenching: either because of strong feedback, or because of the short dynamical time in the central regions of galaxies, which leads to rapid consumption of the cold gas reservoir (e.g. Wang et al. 2019). However, without knowledge of the progenitors of PSB galaxies, it is impossible to establish whether they have always been compact, or if their half-light radii have become smaller as a result of a central starburst and subsequent quenching.

Still, if the second hypothesis is true, we expect high-z PSB galaxies to exhibit clear evidence of a central starburst, such as outwardly-increasing stellar age (as indeed observed in some local PSB galaxies; see e.g. Pracy et al. 2013; Owers et al. 2019; Chen et al. 2019). These inverse gradients are contrary to what is observed in the majority of both SF and $\mathrm{Q}$ galaxies: there is in fact overwhelming evidence that most galaxies form in an inside-out fashion. Firstly, by comparing the size of the star-forming gas disc to the size of the stellar disc, the instantaneous radial growth rate of SF galaxies has been shown to be positive (Pezzulli et al. 2015; Wang et al. 2019; Nelson et al. 2016; Paulino-Afonso et al. 2017; Suzuki et al. 2019). Secondly, the stellar populations of most $\mathrm{SF}$ and $\mathrm{Q}$ galaxies have negative age gradients with radius (e.g. González Delgado et al. 2015; Zibetti et al. 2017): these gradients are qualitatively consistent with the outcome of inside-out growth integrated over cosmic time (Schönrich \& McMillan 2017) ${ }^{1}$. This is also true for individually-measured stars in the Milky Way, both overall (i.e. the bulge is older than the disc, e.g. Valenti et al. 2013) and within the disc itself (Martig et al. 2016).

Measuring age gradients requires high-quality, spatially resolved spectroscopy in the optical rest-frame, but until now these observations at intermediate/high redshift have been out of reach, or limited to small samples (Belli et al. 2017).

The Large Early Galaxy Astrophysics Census (LEGAC; van der Wel et al. 2016) changed this state of affairs: LEGA-C provides the Astrophysics community with deep spectra for $\gtrsim 3000$ galaxies at redshift $z \approx 0.8$, when the Universe was only half its present age. In this work, we leverage the extraordinary depth of LEGA-C to study the structural imprint of inside-out or central-starburst growth in PSB galaxies at roughly half the age of the Universe. After introducing the data and the sample (§ 2), we show that PSB galaxies have distinctive gradients in their Lick indices, different from the control sample (§3), and consistent with an inverse age gradient $(\S 4)$. We conclude this work with a discussion of the implications and with a summary of our findings $(\S 5)$. Throughout this paper, we assume a flat $\Lambda \mathrm{CDM}$ Cosmology with $H_{0}=70 \mathrm{~km} \mathrm{~s}^{-1} \mathrm{Mpc}^{-1}$ and $\Omega_{m}=0.3$ and

1 Contrary to this picture, there is considerable evidence that some Q galaxies have positive or U-shaped age gradients (La Barbera et al. 2012; Zibetti et al. 2020). As it will become clear, this fact by itself does not contradict the picture we present in this paper. 
a Chabrier initial-mass function (Chabrier 2003). All magnitudes are in the AB system (Oke \& Gunn 1983).

\section{DATA ANALYSIS}

\subsection{The LEGA-C Survey}

LEGA-C (van der Wel et al. 2016) is a deep spectroscopic survey targeting $0.6<z<1.0$ massive galaxies in the COSMOS field, using the VIMOS spectrograph (Le Fèvre et al. 2003) on the ESO Very Large Telescope. The LEGA$\mathrm{C}$ primary sample consists of $\approx 3000$ galaxies brighter than $K_{s}=20.7-7.5 \log [(1+z) / 1.8]$, roughly equivalent to a mass-selection limit $\log M_{\star} / \mathrm{M}_{\odot}>10$. Each galaxy was observed for $20 \mathrm{~h}$, reaching a typical signal-to-noise ratio $S N R \approx 20 \AA^{-1}$ in the continuum. The median seeing fullwidth at half-maximum is FWHM $=1.0$ arcsec, sampled with 0.205 arcsec spatial pixels, which is sufficient to extract spatial information (e.g. Bezanson et al. 2018; hereafter we refer to spatial pixels as spaxels and to spectral pixels simply as pixels). What sets LEGA-C apart from previous surveys is its unique combination of ultra-deep data, large sample size and spatial resolution: these three characteristics enable us to study resolved stellar population properties in a statistically meaningful sample.

We use stellar masses $M_{\star}$ measured by fitting the 30 photometric bands of the UltraVISTA catalogue, covering the wavelength range $0.15-24 \mu \mathrm{m}$ (Muzzin et al. 2013). Semi-major axis effective radii $R_{e}$ were measured on $H S T$ ACS F $814 \mathrm{~W}$ images obtained as part of the COSMOS program (Scoville et al. 2007), using GALFIT (Peng et al. 2010) and the procedure of van der Wel et al. (2012). Spectroscopic redshifts were obtained by fitting the galaxy spectra with a library of synthetic stellar population models (Conroy et al., in prep.), following the procedure highlighted in Bezanson et al. (2018). Rest-frame $U-V$ and $V-J$ colours were calculated by fitting a set of seven template spectra to the UltraVISTA SED photometry, (see Straatman et al. 2018, for a full description).

\subsection{Sample selection}

Our parent sample is taken from the LEGA-C public Data Release 2 (Straatman et al. 2018), selected to have $f_{\text {use }}=1$ (1462 galaxies; see Straatman et al. 2018 for the definition of the quality flag $f_{\text {use }}$ ), with four or more radial bins with $S N R>10$ pixel $^{-1}$ each (see $\S 2.3$; 614 galaxies), and with $R_{e}>0.5 \times(\mathrm{FWHM} / 2.355)$, where the seeing FWHM was measured directly on the slit images of each galaxy, using HST photometry as unconvolved reference (van Houdt in prep.; 603 galaxies). We identify passive galaxies in this sample using the $U-V$ vs $V-J$ colour-colour diagram (cf. Labbé et al. 2005, van der Wel et al. 2016; 298 galaxies), and we select 17 PSB galaxies ${ }^{2}$ as having a median index over the spatial measurements $\mathrm{H} \delta_{\mathrm{A}} \geq 4 \AA$, corresponding to an approximate simple-stellar-population-age of $1-1.5 \mathrm{Gyr}$, and typically adopted as selection threshold

2 The total was 19 galaxies, but we further discarded two targets contaminated by interlopers. for spectroscopically-selected PSB galaxies (Wu et al. 2018) ${ }^{3}$ The combination of colour and absorption-strength selection criteria is robust against contamination from dust-obscured starbursts (see e.g. Wu et al. 2018, and Dressler et al. 1999; Poggianti et al. 1999). Other authors have used a cut on inclination (Pawlik et al. 2018), but we find that inclination does not drive our results, hence no inclination cut has been applied (see §3.1). These PSB galaxies consist of six centrals, six isolated, three satellites and two where no environment could be assigned (Darvish et al. 2017). As such, we can exclude that this sample is dominated by satellites, or subject to ram-pressure stripping. As a control sample, we take 141 passive galaxies having the same mass range as the PSB sample, but median $\mathrm{H} \delta_{\mathrm{A}}<4 \AA$. Choosing a stricter cut in $\mathrm{H} \delta_{\mathrm{A}}$ does not change the properties of the control sample, because the bulk of the control galaxies have $\mathrm{H} \delta_{\mathrm{A}}$ well below $4 \AA$ (only 14 galaxies have median $\mathrm{H} \delta_{\mathrm{A}}$ between 2.5 and $4.0 \AA$ ). Even though a control sample having the same mass distribution as the PSB sample would be better suited to control for mass-related biases, in practice such selection is not possible with our data (see $\S 3.1$ for a discussion).

The position of the PSB galaxies on the mass-size plane is illustrated in Fig. 1, where each target is represented by its $H S T$ image, so that each inset is placed at the approximate location of the galaxy portrayed (each inset was allowed a maximum offset of $0.2 \mathrm{dex}$ in $\log M_{\star}$ and $0.1 \mathrm{dex}$ in $\log R_{e}$, to avoid overlappings).

We use HST imaging to assign to each galaxy a probability that it underwent a recent merger. Three astronomers visually inspected the galaxies and the residuals of the bestfit GALFIT models, looking for two merger signatures: tidal features and double cores. Notice that close neighbours are not classified as mergers, unless tidal features are visible either in the HST image or in the residuals. Galaxies were classified as either mergers (score of 1 ) or non-merger (score of 0 ). The average score is the probability that a given target is a merger remnant. Galaxies with a score $P$ (merger) $\geq 0.5$ are highlighted by insets with solid black contours in Fig. 1. For PSB galaxies, we find $7 / 17$ or $40 \%$ of mergers.

Galaxies with/without detectable merger signatures have consistent values of the integrated $\mathrm{H} \delta_{\mathrm{A}}\left(\right.$ mean $\left\langle\mathrm{H} \delta_{\mathrm{A}}\right\rangle=$ $5.91 \pm 0.45 \AA$ and $5.49 \pm 0.28 \AA$ respectively) and Fe4383 $($ mean $\langle\mathrm{Fe} 4383\rangle=1.75 \pm 0.36 \AA$ and $2.34 \pm 0.37 \AA$ respectively). However, we find that galaxies with integrated $\mathrm{H} \delta_{\mathrm{A}}$ larger than the median value $\left(\mathrm{H} \delta_{\mathrm{A}} \geq 5.54 \AA\right.$ ) , have somewhat smaller size than galaxies with $\mathrm{H} \delta_{\mathrm{A}}<5.54 \AA$ ), but the significance is only two standard deviations. Still, the direction of this anti-correlation between $\mathrm{H} \delta_{\mathrm{A}}$ and half-light radius is the same reported in $\mathrm{Wu}$ et al. (2020) for a larger PSB sample. Nevertheless, we find that splitting the sample at $P$ (merger) $\geq 0.5$ or at the median value of the half-light radius does not change our results, apart from lowering their statistical significance $(\S 3.1)$.

3 The selection is almost unchanged (one galaxy is changed) if we use the value of the integrated $\mathrm{H} \delta_{\mathrm{A}}$, as expected from the excellent agreement between the median and integrated index measurements (see Fig. 3). Moreover, we note that our results are qualitatively unchanged if we use a stricter selection threshold at $\mathrm{H} \delta_{\mathrm{A}} \geq 5 \AA$. 


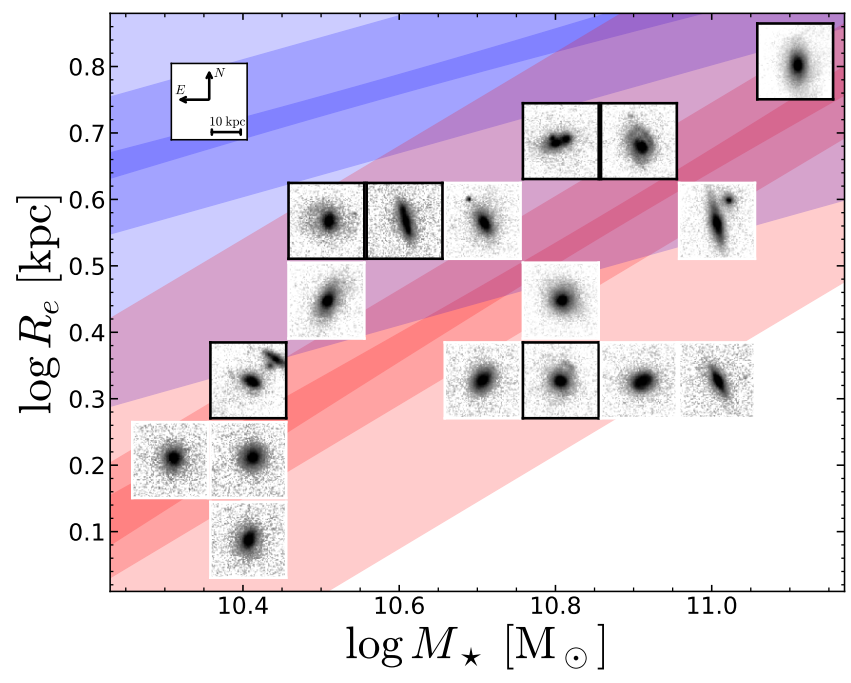

Figure 1. Our sample of post-starburst galaxies has a range of masses, sizes and morphologies, including $40 \%$ of mergers. Galaxies with $P$ (merger) $\geq 0.5$ are highlighted using solid black contour insets. Each image is a $10 \times 10 \operatorname{arcsec}^{2}$ cutout from HST ACS $\mathrm{F} 814 \mathrm{~W}$, and the inset centre is placed at the approximate location on the mass-size plane of the portrayed galaxy (offsets of up to 0.2 dex are allowed for display purposes). The orientation and median physical scale of the images is indicated in the top left inset. The red (blue) transparent regions indicate the best-fit linear model to the mass-size relation for quiescent (star-forming) galaxies; from darkest to lightest, the regions highlight the $95 \%$ confidence interval, the $68 \%$ prediction interval and the $95 \%$ prediction interval. The best-fit parameters for the mass-size relations were derived using the least-trimmed squares algorithm (Rousseeuw \& Driessen 2006; Cappellari et al. 2013). Despite our spatial resolution constraint, which systematically selects the largest PSB galaxies, the sample lies appreciably below the star-forming masssize relation for LEGA-C

\subsection{Spatially resolved Lick index measurements}

We measure Lick indices, defined as in Worthey \& Ottaviani (1997) and Trager et al. (1998), as well as the $\mathrm{D}_{\mathrm{n}} 4000$ index (Balogh et al. 1999). The method we use, developed by Scott et al. (2017) and Barone et al. (2020), can be thought of as a non-parametric emission-line subtraction. The goal of their algorithm is to leverage spectral information away from emission-line regions to reconstruct the galaxy stellar spectrum inside such regions. Empirical stellar spectra have been shown to encode significantly more information per spectral element compared to synthetic spectra (e.g. Martins \& Coelho 2007; Plez 2011), therefore they are more likely to accurately reproduce the observed galaxy spectra (e.g. van de Sande et al. 2017, their fig. 25), and to capture the necessary information to reconstruct the spectrum in the masked regions. For this reason, we fit the LEGA-C spectra with an empirical stellar template library. We use the MILES stellar template library because of its generous range in stellar classes (Sánchez-Blázquez et al. 2006; Falcón-Barroso et al. 2011), but we obtain equivalent results using the high spectral resolution ELODIE library (Prugniel \& Soubiran 2001).

We fit the stellar continuum using the penalised Pixel Fitting code PPXF (Cappellari \& Emsellem 2004), following the procedure developed by Scott et al. (2017). In short, we fit the spectrum optimising for the template weights, for the first and second moment of the line-of-sight velocity distribution, $v$ and $\sigma$, and for a $12^{\text {th }}$-order additive polynomial. ${ }^{4}$

The fit is performed in three iterative steps. The first iteration is used to estimate the noise spectrum; the second iteration is used to identify weak emission lines and bad pixels and the third and final gives the best-fit parameters. The spectrum in bad pixels and in regions of line emission is replaced by the best-fit stellar spectrum. This step is especially important for the Balmer absorption indices, which overlap regions of nebular emission. Whether the higherorder Balmer lines are masked or not does not affect our results ( $\mathrm{H} \epsilon$ and bluer; Barone et al. 2020).

Once the emission-line corrected spectra are determined, each index is measured after convolving the spectrum with a Gaussian, so that the final spectral resolution matches the spectral resolution of the relevant index. For more details on the fitting and measurement procedure refer to Scott et al. (2017).

In order to guarantee an acceptable precision, we bin the slit spectra out from the central spaxel to guarantee a $\mathrm{SNR}=10 \mathrm{pixel}^{-1}$. Firstly we fit the individual spectra, to estimate their SNR. We then fold the slit about the central spaxel, ranking the spectra by their distance to the centre. Starting from the central spaxel, we create spatial bins by summing adjacent spaxels until the target SNR is met. For galaxies with obvious contamination, we consider only the half of the spectrum away from the companion or interloper object. Two example fits are shown in Fig. 2.

For our analysis, we focus on two indices: $\mathrm{H} \delta_{\mathrm{A}}$ and Fe4383. There are two reasons for this choice: firstly, it represents a minimal index set that is able to break the agemetallicity degeneracy, at least for the age range relevant to PSB galaxies. In particular, $\mathrm{H} \delta_{\mathrm{A}}$ has a local maximum for $\mathrm{a} \approx 0.3 \mathrm{Gyr}$-old simple stellar population (Worthey \& Ottaviani 1997; Kauffmann et al. 2003), so that it is not possible to invert the age- $\mathrm{H} \delta_{\mathrm{A}}$ function using $\mathrm{H} \delta_{\mathrm{A}}$ alone. However, for the ages and metallicities relevant to this work, adding Fe4383 allows to break this age degeneracy (see §4.1). Secondly, these indices ensure uniform coverage across the largest possible sample, whereas indices defined at redder wavelengths drop out of the LEGA-C observed range with increasing redshift.

Each index measurement has a measurement uncertainty, derived from the residuals of the spaxel spectrum with respect to the best-fit spectrum. We find that these values are underestimated and we derive an upscaling factor as follows. Given the relatively high SNR in the central spaxels, we often have two measurements at a given distance from the centre, one for each side of the slit. We assume that galaxies are symmetric about their centre, so we can use

4 Even though this choice is motivated by convergence criteria (i.e. higher degree polynomials do not exhibit faster variation with wavelength), we find that the value of the Lick indices does not change for degrees higher than 2. This behaviour is due to a combination of the local nature of the Lick measurements and of our good data quality. Replacing additive polynomials with multiplicative polynomials does not change our results either (see also Bezanson et al. 2018). 


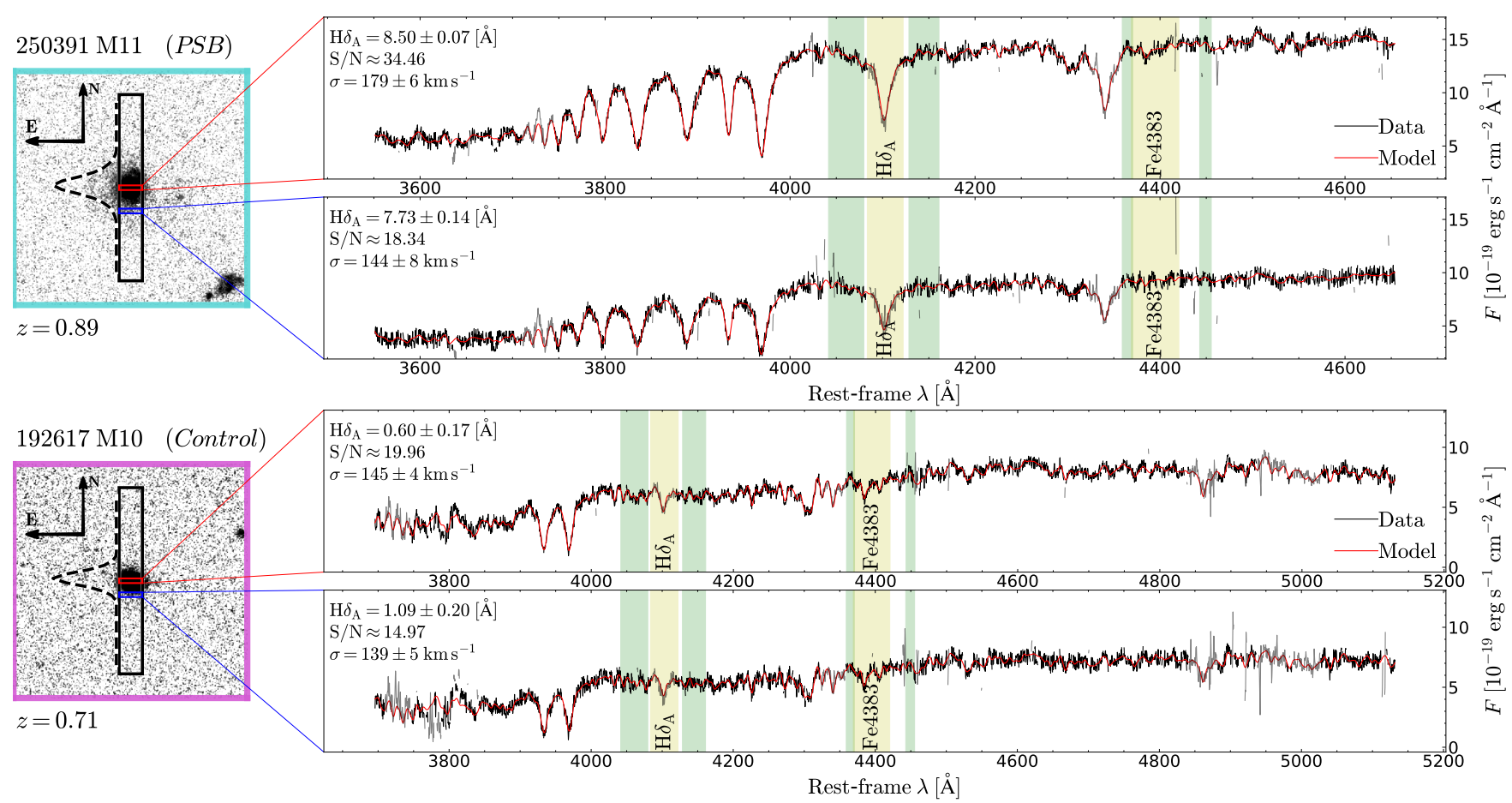

Figure 2. Central and outer rest-frame spectra for the post-starburst galaxy M11.250391 (top) and for the quiescent galaxy M10.192617 (bottom). The continuum and the index regions for the Lick indices $\mathrm{H} \delta_{\mathrm{A}}$ and $\mathrm{Fe} 4383$ are highlighted in green and yellow respectively. The approximate spaxel position for each spectrum is marked on the finding chart. For the quiescent galaxy, $\mathrm{H} \delta_{\mathrm{A}}$ is higher in the outskirts than in the centre, for the PSB galaxy $\mathrm{H} \delta_{\mathrm{A}}$ is strongest in the centre. The black lines represent LEGA-C data, whereas the red lines are the PPXF best-fit models (see $\S 2.3$ for more details). Regions of the spectrum where the data were masked are rendered in grey: these regions are excluded either because they fail a three $\sigma$ clipping threshold, or because of possible emission lines (regardless of whether line emission has been detected).

the difference between the two measurements to rescale the formal uncertainties on our measurements. Comparing the measurements from either side of the galaxies, we find no systematic offset, but the standard deviation is larger than the formal uncertainties. We rescale the formal uncertainties by a factor that depends on the Lick index being considered and on the value of the SNR. Given the SNR depends strongly on the distance from the centre of each galaxy, our SNR rescaling factors are effectively a function of radius. For $\mathrm{H} \delta_{\mathrm{A}}$, the factor ranges from 1 (at the highest SNR) to 3 (for $10<S N R<15$ pixel $^{-1}$ ). For Fe4383, the factor ranges from 1 to 2.5. Similar results were obtained following the method of Straatman et al. (2018), i.e. using repeat observations of 61 galaxies to assess the random uncertainties on the Lick index measurements. The main difference with our method is that using repeat observations tends to overestimate the uncertainty for PSB galaxies, which have systematically stronger $\mathrm{H} \delta_{\mathrm{A}}$ absorption compared to the sample of repeat observations (only one PSB galaxy has two observations).

In Fig. 3 we compare the value of the integrated Lick indices from Straatman et al. (2018) to the (unweighted) median value for our resolved measurements. We show separately the PSB and control sample as cyan stars and magenta circles, but we fit a single relation to both sets, and find excellent agreement between the two measurements. For $\mathrm{H} \delta_{\mathrm{A}}$ we find a best-fit linear slope of $0.96 \pm 0.03$ and a rootmean square residual along the y-axis of $0.33 \AA$ (panel a). Thus the best-fit relation is statistically consistent with the

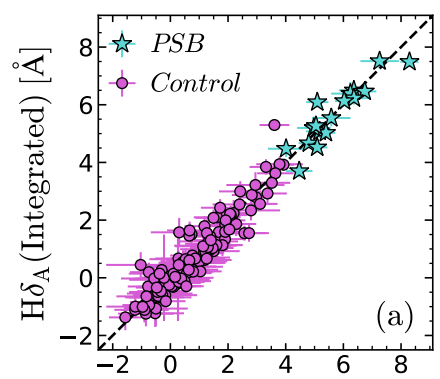

$\left\langle\mathrm{H} \delta_{\mathrm{A}}(\right.$ Resolved $\left.)\right\rangle[\AA]$

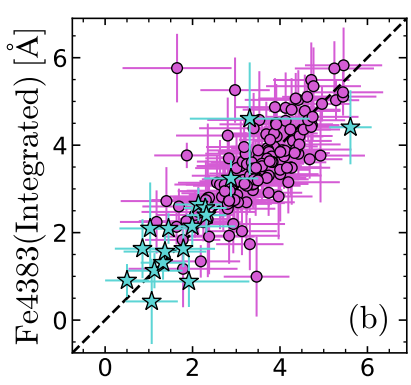

$\langle$ Fe4383(Resolved) $\rangle[\AA]$
Figure 3. We find very good agreement between the median of the spatial measurements and the measurement on the integrated slit profile, for both $\mathrm{H} \delta_{\mathrm{A}}$ and $\mathrm{Fe} 4383$ (panels a and b respectively). The cyan stars are PSB galaxies, the magenta circles are the control sample of quiescent galaxies (the errorbars have been rescaled). The best-fit relations have slopes $0.96 \pm 0.03$ (for $\mathrm{H} \delta_{\mathrm{A}}$ ) and $1.03 \pm 0.08$ (for Fe4383), consistent with unity. The observed scatter about the best-fit relations are $\Delta=0.26$ and $\Delta=0.52$ respectively, consistent with the measurement uncertainties (after rejecting $3-\sigma$ outliers, the reduced $\chi^{2}$ value is 1.01 in both cases).

identity. As for the scatter, if we assume that the precision of the two determinations is the same and that there was no intrinsic scatter (due e.g. to systematic errors), we can estimate the average measurement uncertainty as $0.26 / \sqrt{2}=0.18 \AA$. Mutatis mutandis, similar considerations 
apply to Fe4383; here the best-fit linear relation has slope $1.03 \pm 0.08$ and observed scatter 0.51 (panel b). In principle, a comparison between the unweighted median and the integrated indices is biased, because the latter are de facto fluxweighted. However, if we repeat the above comparison after replacing the unweighted median indices with the inverseerror weighted indices, the results are statistically consistent with what we have reported for the unweighted median (except for the best-fit slope of the $\mathrm{H} \delta_{\mathrm{A}}$ relation, which goes from $0.96 \pm 0.03$ to $1.09 \pm 0.02$ ). This consistency is probably due to the mix of PSB and non-PSB galaxies, because, as we will argue in the next section, these two sets have different radial properties. These properties are likely to impart opposite biases on the unweighted median indices compared to the integrated indices. Galaxy 107643 M4 is the most prominent outlier in Fig. 3 (more than three standard deviations), but it has a relatively bright interloper that might affect the integrated spectrum. This galaxy is part of the control sample, and its inclusion or removal does not change the outcome of our analysis.

When we repeat our analysis with the ELODIE stellar template library, we find excellent agreement in the average value of the indices: considering only spectra with $\mathrm{SNR} \geq$ 10 pixel $^{-1}$, we find no mean offset in either $\mathrm{H} \delta_{\mathrm{A}}$ and $\mathrm{Fe} 4383$ $\left(\Delta \mathrm{H} \delta_{\mathrm{A}}=-0.001 \pm 0.002 \AA\right.$ and $\left.\Delta \mathrm{Fe} 4383=0.012 \pm 0.009 \AA\right)$.

\section{RESULTS}

In Fig. 4a we show the average radial trends of $\mathrm{H} \delta_{\mathrm{A}}$ relative to the central value, for both PSB galaxies (cyan) and the control sample (magenta). The radial profiles of individual galaxies have been binned in $R_{e}$, with the lines tracing the (moving) inverse-variance weighted median. The uncertainty on the median is encompassed by the shaded region (estimated as the semi-difference between the inversevariance-weighted $16^{\text {th }}$ and $84^{\text {th }}$ percentiles, divided by the square root of the number of measurements in each bin). The dashed lines enclose the $16^{\text {th }}$ and $84^{\text {th }}$ percentiles of the distribution. The control sample of quiescent, non-PSB galaxies and the PSB sample have opposite radial trends: the control sample has a weak positive $\mathrm{H} \delta_{\mathrm{A}}$ gradient. In contrast, PSB galaxies have on average decreasing $\mathrm{H} \delta_{\mathrm{A}}$ profiles, i.e. the $\mathrm{H} \delta_{\mathrm{A}}$ index is highest in the central regions and lowest in the outskirts. For the Fe4383 index (Fig. 4b), we find that PSB galaxies have a radially-increasing profile, whereas the control sample has decreasing Fe4383 with radius. Similar results are obtained for other empirical spectral indices, which we do not show for brevity: for example, PSB galaxies have decreasing $\mathrm{H} \gamma_{\mathrm{A}}$ and flat $\mathrm{D}_{\mathrm{n}} 4000$, whereas control galaxies have increasing $\mathrm{H} \gamma_{\mathrm{A}}$ and decreasing $\mathrm{D}_{\mathrm{n}} 4000$ (see Appendix A).

If we assume that PSB galaxies have flat $\mathrm{H} \delta_{\mathrm{A}}$ gradients, we can calculate the probability of measuring by chance a negative gradient as follows: for each radial measurement, we take the distance between the median $\mathrm{H} \delta_{\mathrm{A}}$ and zero (the value expected from a flat gradient; this is equal to $\Delta \mathrm{H} \delta_{\mathrm{A}}$ ). We then divide this distance by the uncertainty on $\Delta \mathrm{H} \delta_{\mathrm{A}}$, and calculate the resulting one-tailed probability of a value exceeding the measurement (we assume a Gaussian distribution). The number of independent radial measurements in the stacked profiles is difficult to calculate, because each

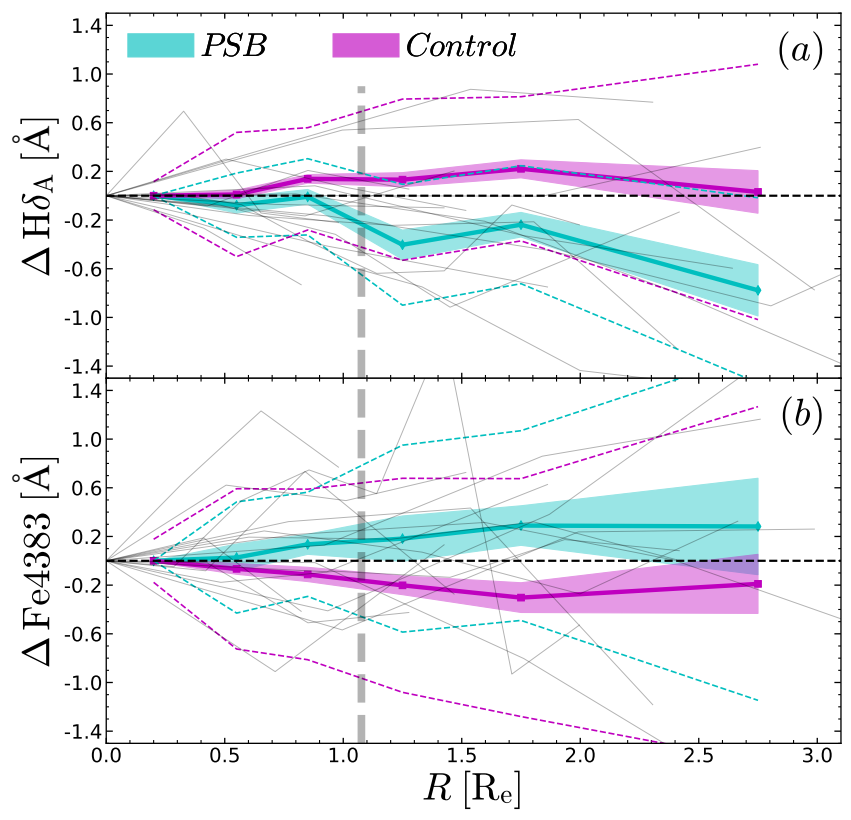

Figure 4. Unlike control galaxies (magenta), post-starburst galaxies (cyan) show decreasing $\mathrm{H} \delta_{\mathrm{A}}$ and flat $\mathrm{Fe} 4383$, clear signatures of a central starburst. The solid lines trace the running median of our measurements, the uncertainties about the median are enclosed by the shaded regions, whereas the coloured dashed lines enclose the $16^{\text {th }}$ and $84^{\text {th }}$ percentiles of the data. The vertical dashed lines marks the $\sigma$-equivalent of the median seeing. Thin grey lines trace the profile of individual PSB galaxies, showing that besides the average trend, individual galaxies present a range of radial profiles (individual control galaxies are not shown). The observed PSB trends are highly significant: in the most conservative estimate, the probability of a false-positive is $P=10^{-4}$.

galaxy has different size and slightly different seeing. We therefore provide the results for the most conservative case only, i.e. assuming only two independent radial measurements. Assuming PSB galaxies have flat $\mathrm{H} \delta_{\mathrm{A}}$ radial profiles, the probability of finding by chance a negative gradient is $P=10^{-4}$. For the Fe4383 gradients, using the same assumptions we get $P=0.04$. Bootstrapping $75 \%$ of our data yields $P=0.06$ and $P=0.04$ respectively. Similarly, the probabilities that the control sample has flat profiles for $\mathrm{H} \delta_{\mathrm{A}}$ and Fe4383 are $P=0.02$ and $P=0.07$ respectively (bootstrapping yields $P=10^{-3}$ and $P=10^{-4}$ ). Combining these opposite trends naturally leads to even smaller probabilities that PSB and control galaxies have the same profiles: $P=10^{-5}$ and $P=0.01$ respectively, for $\mathrm{H} \delta_{\mathrm{A}}$ and $\mathrm{Fe} 4383$ (bootstrapping yields $P=0.03$ and $P=0.01$ ). Assuming three independent radial bins yields $P$-values that are $5-10$ times smaller.

\subsection{Caveats}

The results are qualitatively unchanged if we measure the distance along the slit in physical units; however, since physical units may compound radial trends within galaxies with size trends between galaxies, they are not considered here.

We remark that the extent of our measurements is comparable to the FWHM of the atmospheric seeing (the verti- 
cal dashed line in Fig. 4 is the seeing equivalent $\sigma$, defined as $\mathrm{FWHM} / 2.355$, see $\S 2.2$ ). The value shown is the median seeing for the 17 PSB galaxies, expressed in units of the galaxy $\mathrm{R}_{\mathrm{e}}$, and falls approximately at one $\mathrm{R}_{\mathrm{e}}$. For this reason, the gradients measured here are much flatter than the intrinsic gradients $(\S 4)$, in agreement with what is observed for PSB galaxies at $z \approx 0.1$ (Pracy et al. 2013). The fact that the seeing is comparable to the median effective $R_{e}$ of our PSB sample might explain the change in slope around $R \approx 2 \mathrm{R}_{\mathrm{e}}$, but we cannot exclude the presence of a size-dependent bias for this bin (only 13/17 measurements for the PSB sample, only 36/141 measurements for the control sample).

Due to the small sample size, we are unable to study the relation between inverse gradients and other galaxy properties. However, we find that the trend is qualitatively unchanged if we consider mergers and non-mergers separately, indicating that our results are not driven by prominent morphologic asymmetries. The same is true if we split the PSB sample in two at the median value of the Sérsic index $(0.6 \leq n \leq 6$; the median is 2.9$)$, or at the median value of the apparent effective radius $\left(0.2 \leq R_{e} \leq 0.7\right.$; the median is $0.3 \mathrm{arcsec}$ ), or at the median value of stellar mass $\left(10^{10.3} \leq M_{\star} \leq 10^{11.2}\right.$; the median value is $\left.10^{10.68} \mathrm{M}_{\odot}\right)$, or at the median value of the axis ratio $(0.21 \leq q \leq 0.94$; the median value is 0.64 ). Finally, we repeat the analysis limiting the selection to PSB classified as central or isolated only: the trends are again qualitatively unchanged, ruling out that our results are due to environment effects on satellite galaxies.

In $\S 2.2$, in order to ensure that the study is not limited by the size of the control sample, we selected control galaxies to have the same mass range as the PSB sample. Admittedly, a better choice would be to select control galaxies having the same mass distribution as the PSB sample, because the strength of stellar population gradients of passive galaxies depends on stellar mass and velocity dispersion (e.g. Martín-Navarro et al. 2018; Zibetti et al. 2020). However, the quiescent, non-PSB galaxies that make up the control sample have on average lower SNR and larger measurement uncertainties than PSB galaxies of the same mass. For this reason, imposing the same mass distribution between the PSB and control samples results in too few control galaxies (25) with too large measurement uncertainties to constrain the sample properties. Nevertheless, we find that the massmatched control sample is statistically consistent with the actual control sample used in this work. To further test the effect of mass-dependent bias in the observed gradients, we split the control sample in two subsets at the value of its median stellar mass $10^{10.82} \mathrm{M}_{\odot}$. We find that both subsets have gradients that are statistically consistent with the control sample, but the most-massive half of the sample has steeper Fe4383 gradient than the least-massive half, in qualitative agreement with observations of local galaxies (e.g. Martín-Navarro et al. 2018 find that the most massive elliptical galaxies have steeper radial metallicity gradients). Using either half of the control sample would not change the nature of our results.

Incidentally, the fact that PSB galaxies have opposite radial trends compared to the general population suggests that our results are unlikely to arise from bias due to decreasing SNR with radius.

\section{A TWO-SSP TOY MODEL}

To interpret the observed trends, we implement a sixparameter model to predict the stacked measurements of Fig. 4. As a light profile, we use a one-dimensional Sérsic model, where the spectrum at each radius is the superposition of two simple stellar populations (SSP; i.e. each population has uniform age and metallicity). As SSP spectra we take the MILES models (Vazdekis et al. 2010, 2015), using BaSTI isochrones (Pietrinferni et al. 2004, 2006), so$\operatorname{lar}[\alpha / \mathrm{Fe}]$ and Chabrier IMF (Chabrier 2003). The resulting grid of 636 spectra spans $-2.27<[\mathrm{Z} / \mathrm{H}]<0.40$ and $0.03<$ age $<14.00$ Gyr (replacing BaSTI with Padova isochrones from Bertelli et al. 2009 yields qualitatively consistent results). Our model superimposes two SSPs, representing the central stars and the stars in the outskirts of the galaxy, labelled respectively "in" and "out" (the corresponding SSP parameters are age $e_{i n},[\mathrm{Z} / \mathrm{H}]_{\text {in }}$, age out and $\left.[\mathrm{Z} / \mathrm{H}]_{\text {out }}\right)$. The mass fraction of the "in" SSP to the total is given, at each radius $R$, by:

$f(R)=f_{0} \frac{e^{\frac{R_{m}-R}{R_{d}}}-1}{e^{\frac{R_{m}}{R_{d}}}-1}$

where $R$ is expressed in units of $R_{e}$, and $R_{m}=6 \mathrm{R}_{\mathrm{e}}$ is an arbitrary radius that is "large" relative to the extent of our measurements. $f$ is a declining exponential function, scaled so that the central value is $f(0)=f_{0}$ and downshifted so that $f\left(R_{m}\right)=0$. This choice is motivated as follows. Firstly, stellar generations tend to form superimposed exponential discs (Poci et al. 2019, Buck et al. 2019 - and the ratio of two exponentials is also exponential). Secondly, there is evidence that PSB galaxies host rotation-supported discs (Hunt et al. 2018). The parameter $R_{d}$ specifies the concentration of the central SSP: for any non-negative value, $R_{d}$ is the exponential scale radius of $f(R)$, in the sense that $\partial_{R} f=-f / R_{d}+$ const. (smaller values of $R_{d}$ correspond to more concentrated central populations). As $R_{d} \rightarrow \infty$, we have $f(R) \rightarrow\left(1-R / R_{m}\right)$ : in other words, using Eq. (1) to express $f(R)$ includes both a physically-motivated exponentially-declining fraction, and a linear mixing fraction which represents the simplest uninformed guess. In practice we implement the infinite range in $R_{d}$ by parametrising this scale radius as $\tan \vartheta_{d}$, with $0 \leq \vartheta_{d} \leq \pi / 2$. Thus the fraction $f$ requires two additional parameters: $f_{0}$ and $\vartheta_{d}$. For our purposes, $f_{0}$ and $\vartheta_{d}$ are just nuisance parameters: given (i) our spatial resolution and (ii) the use of a stack analysis, we cannot meaningfully constrain the structure of PSB galaxies, but just the sign of radial gradients of stellar age and metallicity. The Sérsic profile has arbitrary central surface brightness and $R_{e}$, but the Sérsic index is fixed at $n=2.4$, the median value for the PSB sample. The model is convolved with a Gaussian PSF with $\sigma=1 R_{e}$ (see Fig. 4). In summary, our most general model has six free parameters, the age and metallicity for each of the two SSPs, and two more parameters to specify the (monotonic) radial mixing of these two SSPs. The likelihood of the data given these model parameters is expressed as a multivariate Gaussian over the observed $\mathrm{H} \delta_{\mathrm{A}}$ and $\mathrm{Fe} 4383$ measurements. We assume flat priors on all the model parameters, with the allowed range equal to the physical range of each parameter: $0.03<$ age $_{\text {in }}$, age $_{\text {out }}<14.00 \mathrm{Gyr}$, $-2.27 \leq[\mathrm{Z} / \mathrm{H}]_{\text {in }}, \quad[\mathrm{Z} / \mathrm{H}]_{\text {out }} \leq 0.40,0 \leq \vartheta_{d} \leq \pi / 2$ and 


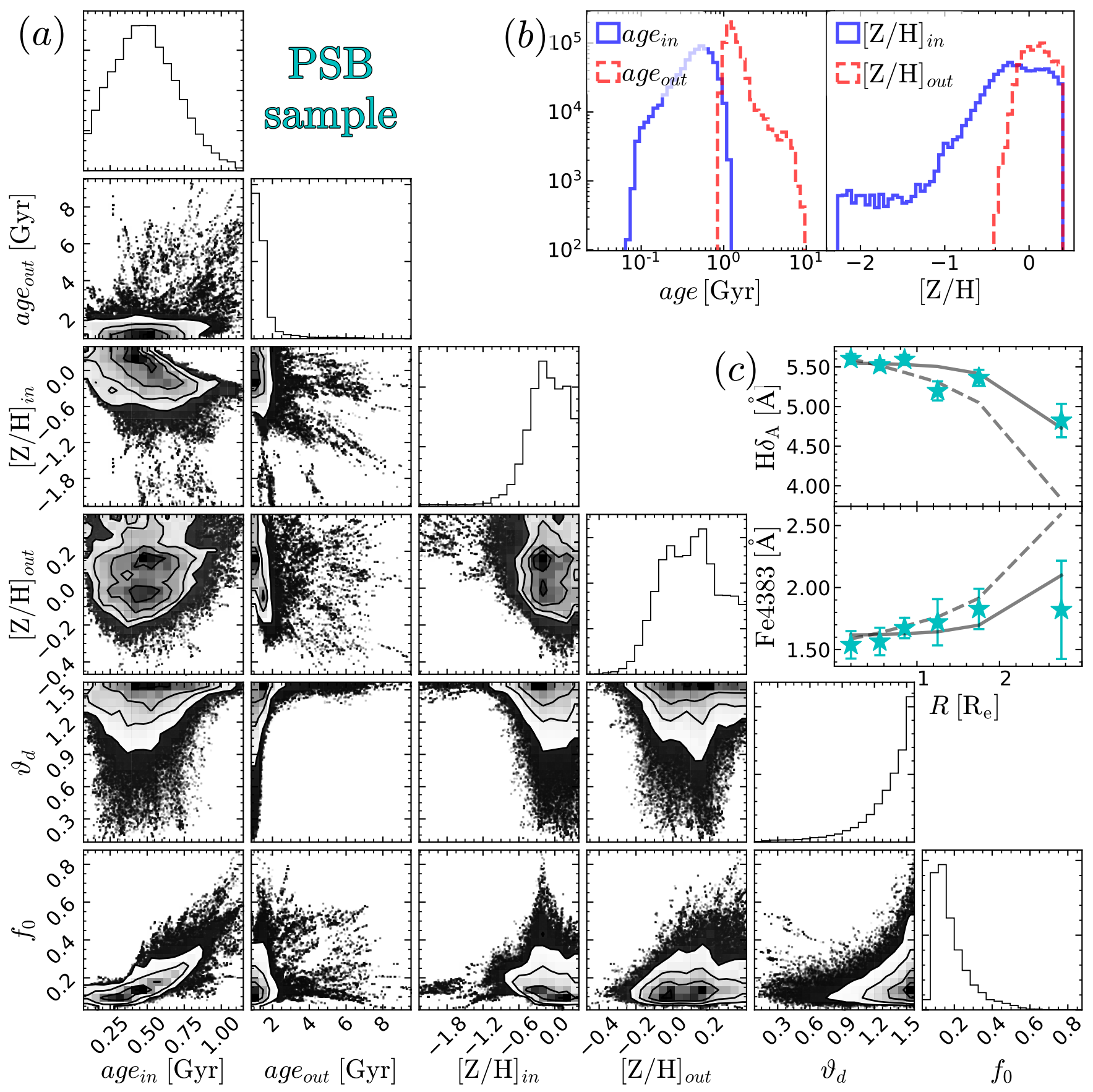

Figure 5. Our two-SSP toy model requires inverse age gradients in PSB galaxies (Panel a). The model uses two SSPs with different spatial distribution to reproduce the median observed gradients in both $\mathrm{H} \delta_{\mathrm{A}}$ and Fe4383 (Panel c). The dashed/solid lines show the model prediction before/after seeing convolution, and the cyan stars trace the median index values for the PSB sample. The age of the inner and outer SSP are clearly different: the inner SSP (solid blue histogram in Panel b) is clearly younger than the outer SSP (dashed red histogram). In contrast, SSP metallicities are consistent within the uncertainties. The corner diagram shows the marginalised probability for the model; the strongest correlation is between $f_{0}$ (tracing the burst fraction) and age $e_{\text {in }}$ (tracing the burst age).

$0 \leq f_{0} \leq 1$. We combine the likelihood and priors to write the posterior distribution (apart from the evidence), and we estimate the model parameters by integrating the posterior distribution with the Markov Chain Monte Carlo approach (Metropolis et al. 1953).

Constraining this six-parameter model using twelve measurements is problematic, but our goal is not to infer an accurate value of the parameters. We use the model as a benchmark, to assess the plausibility of less general submodels, obtained by constraining some of the six parameters from the general model, and we show that any acceptable solution has properties that are inconsistent with the properties of the control sample. 


\subsection{The benchmark model for PSB galaxies}

The results for the most general PSB model are shown in Fig. 5. Panel a shows the posterior distribution for the six model parameters, marginalised over all possible sets of four and five parameters. A summary of the model results is reported in Table 1. For ease of comparison, the posterior distributions of age $_{\text {in }}$ and age $_{\text {out }}$ and of $[\mathrm{Z} / \mathrm{H}]_{\text {in }}$ and $[\mathrm{Z} / \mathrm{H}]_{\text {out }}$ are reported also in panel b (using a logarithmic scale for age). Panel c compares the measured Lick indices (cyan stars with errorbars) to the prediction of the most likely model (i.e. the mode of the posterior distribution); the dashed and solid grey lines trace respectively the intrinsic profile and the seeing convolved profile. Within one $R_{e}$, the intrinsic $\mathrm{H} \delta_{\mathrm{A}}$ gradient for our stacked profile is $\approx-0.76 \pm 0.03 \AA / R_{e}$, consistent with the median value for local PSB galaxies $\left(-0.83 \pm 0.23 \AA / R_{e}\right.$; Chen et al. 2019. We considered both their "central" and "ring-like" PSBs to calculate the median, consistent with our sample selection criteria that do not differentiate between different PSB morphologies. The fit was performed using weighted least squares optimization.)

The corner plot shows the well-known age-metallicity degeneracy (Worthey 1994), for each of the two SSPs independently. The Spearman rank correlation coefficient is $\rho=-0.39$ for age $_{\text {in }}$ and $[\mathrm{Z} / \mathrm{H}]_{\text {in }}$ and is $\rho=-0.52$ for age out and $[\mathrm{Z} / \mathrm{H}]_{\text {out }}$ (panel a; all $P$-values are zero owing to the large number of sample points). Of all the possible parameter pairs, we find the strongest degeneracy between $f_{0}$ and age $_{\text {in }}(\rho=0.84)$; this can be interpreted as the degeneracy between the burst fraction (governed by the central value $f_{0}$ ) and the burst age (Serra \& Trager 2007).

With these degeneracies in mind, we can inspect panel $\mathrm{b}$, which reports the age and metallicity histograms of the two SSPs on the same scale: here the central SSP (solid blue histogram) has both younger age and lower metallicity than the outer SSP (red dashed histogram). For SSP age, we find age $_{\text {in }}=0.48_{-0.21}^{+0.23} \mathrm{Gyr}$ and age $e_{\text {out }}=1.28_{-0.20}^{+0.43}$ Gyr (here and in the following the results quoted refers to the $50^{\text {th }}$ and the uncertainties encompass the $16^{\text {th }}$ and $84^{\text {th }}$ percentiles of the relevant posterior distribution). For metallicity, the results are $[\mathrm{Z} / \mathrm{H}]_{\text {in }}=-0.13_{-0.22}^{+0.33}$ and $[\mathrm{Z} / \mathrm{H}]_{\text {out }}=0.10_{-0.06}^{+0.17}$. We find that metallicity is not well constrained, as expected from young SSPs. However, while the difference in $[\mathrm{Z} / \mathrm{H}]$ is not statistically significant (within one standard deviation), the age difference is larger than 3.5 standard deviations: the probability $P$ that the two SSPs have the same age is $P<2 \times 10^{-4}$ (this value assumes a Gaussian distribution and is the most conservative result; using the marginalised posterior distribution we obtain $\left.P<1.3 \times 10^{-6}\right)$. The strong separation between the age of the two SSPs is mostly due to the sharp cutoff in the posterior distribution of age out below $\approx 0.9 \mathrm{Gyr}$ (red dashed age histogram in panel b). This strong cutoff may be surprising, because $\mathrm{H} \delta_{\mathrm{A}}$ has a local maximum at $0.1-1 \mathrm{Gyr}$ (the exact value depends on metallicity, see e.g. Worthey \& Ottaviani 1997 and Kauffmann et al. 2003, their fig. 2), so that there is a strong degeneracy between $\mathrm{H} \delta_{\mathrm{A}}$ and SSP age precisely where the model infers a cutoff in the distribution. The solution to this apparent conundrum is in the value of Fe4383: at fixed metallicity, Fe4383 increases with SSP age. For this reason, even though age out younger than $0.9 \mathrm{Gyr}$ could indeed explain the decreasing values of $\mathrm{H} \delta_{\mathrm{A}}$ with radius, it would also predict a radially decreasing Fe4383, opposite to what is observed. In addition, even for the highest metallicity, SSPs young enough to have $\mathrm{H} \delta_{\mathrm{A}} \lesssim 4 \AA$ have $\mathrm{Fe} 4383<0 \AA$, inconsistent with the observations. Notice also that while $a_{g e} e_{\text {in }}<a g e_{\text {out }}$, we do not find an "old" outer SSP: this is due to the use of a single SSP instead of an extended SFH, as we show in $\S 4.4$. We conclude that this model, simple yet general, strongly prefers an age gradient over a metallicity gradient to explain the median index profiles observed in our PSB sample.

\subsection{Modelling the control sample}

We have already ruled out the possibility that the median gradient of the PSB and of the control sample are the same. But could these different gradients arise from similar stellar populations, observed at different ages? This question is paramount to understanding whether (in an average sense) the control sample is consistent with passive evolution of the PSB sample. To give an answer, we apply our toy model to the median Lick profiles of the control sample. The results are illustrated in Fig. 6, where the meaning of the symbols are the same as in Fig. 5. The model predictions are compared to the data in panel c: within one $R_{e}$, we find a $H \delta_{A}$ gradient of $\approx 1 \AA / R_{e}$, whereas outside one $\mathrm{R}_{\mathrm{e}}$ the intrinsic index profile is flat. This behaviour results from the combination of a relatively high central fraction (see the posterior probability of $f_{0}$ in panel a) and compact spatial distribution (small $\vartheta_{d}$ ). This behaviour however may not be robust, given the strong degeneracy between $f_{0}$ and $\vartheta_{d}$, which affects the intrinsic radial gradients $(\rho=-0.76)$. We also remark that the last radial measurement appears to be an outlier: ignoring this point yields a less steep gradient of $\approx 0.8 \AA / R_{e}$.

Examining the corner diagram (panel a), we find again that age and metallicity anticorrelate for each $\operatorname{SSP}(\rho=$ -0.10 and -0.31 for the inner and outer SSP, respectively). However, compared to the posterior probability distribution for the PSB sample, for the control sample these degeneracies are significantly smaller (in absolute value). On the other hand, we find a strong correlation of the metallicity of the inner SSP $\left([\mathrm{Z} / \mathrm{H}]_{i n}\right)$ with both age and metallicity of the outer SSP: we find $\rho=0.61$ for age out and $\rho=-0.72$ for $[\mathrm{Z} / \mathrm{H}]_{\text {out }}$. These strong correlations are likely due to metallicity being the strongest driver of both $\mathrm{H} \delta_{\mathrm{A}}$ and $\mathrm{Fe} 4383$ for old stellar populations; their presence highlights the need for comprehensive modelling in order to interpret our data.

Panel b shows the age and metallicity histograms of the two SSPs: unlike for PSB galaxies, here the central SSP (solid blue histogram) has both older age and higher metallicity than the outer SSP. We find age $e_{i n}=8.3_{-4.2}^{+3.9} \mathrm{Gyr}$ whereas age $e_{\text {out }}=2.27_{-0.05}^{+0.08} \mathrm{Gyr}$; for metallicity, the results are $[\mathrm{Z} / \mathrm{H}]_{\text {in }}=0.21_{-0.19}^{+0.13}$ and $[\mathrm{Z} / \mathrm{H}]_{\text {out }}=-0.15_{-0.04}^{+0.04}$. For control galaxies, the probability that the two ages are the same is $P<3 \times 10^{-4}$ (using the joint posterior probability distribution of age $_{\text {in }}$ and age $\left.e_{\text {out }}\right)^{5}$ It is unclear whether and to

5 In this case, assuming a Gaussian probability with standard deviation equal to the standard deviation of the posterior yields $P<0.1$, but this estimate is too conservative: the large difference in probability between the two estimates arises from the shape of the posterior probability distribution of $a g e_{i n}$, that has a sharp cutoff below $\approx 2.5$ Gyr (Fig. 6a). 
what extent this sharp cutoff might be caused by our stacking analysis. In fact, on one hand the youngest galaxies are (on average) the smallest, which enhances their contribution to the innermost radial bins and biases age $e_{i n}$ to younger values; on the other hand, however, the oldest galaxies, despite being on average the largest, tend to have the steepest light profiles, which also enhances their contribution to the innermost bins. Disentangling these two competing effects is however beyond the scope of this paper. From Fig. 6b, we also notice that SSP age for both the inner and outer SSPs is larger than in PSB galaxies. This might be due either to the different mass distribution of the two samples, but also to problems inherent with the stacking analysis. For example, if the youngest PSB galaxies are also the most compact, their PSF would be larger than the median PSF (when expressed in units of $R_{e}$ ), causing more contamination between the inner and outer SSP. In light of this ambiguity, we do not overinterpret the observed age difference between control galaxies and the outer SSP of PSB galaxies. For metallicity, we find $[\mathrm{Z} / \mathrm{H}]_{\text {in }}>[\mathrm{Z} / \mathrm{H}]_{\text {out }}$, but this result is not statistically significant (the probability that the two SSPs have the same metallicity is $P<0.07)$.

At face value, however, we find that modelling the control sample requires the central SSP to be older and more metal rich than the outer SSP, as observed in most local quiescent galaxies (e.g. McDermid et al. 2015; Zibetti et al. 2020; Ferreras et al. 2019) and at variance with the model for PSB galaxies.

\subsection{Constrained PSB models}

Could an inside-out SSP gradient reproduce the observed radial trends of $\mathrm{H} \delta_{\mathrm{A}}$ and $\mathrm{Fe} 4383$ for PSB galaxies? We have already shown that the benchmark model points to an inverse age structure for PSB galaxies, therefore we know that an inside-out structure is less likely. However, our two-SSP model has only six degrees of freedom, therefore its predictive power is modest. For this reason, it is important to evaluate directly how much worse an inside-out model would be relative to the benchmark model. To address this question, we create two more PSB models, identical to the benchmark model, but constrained to have inside-out age or metallicity gradients. The first model has free age but $[\mathrm{Z} / \mathrm{H}]_{\text {in }}>[\mathrm{Z} / \mathrm{H}]_{\text {out }}$ (PSB inside-out metallicity model, Table 1). For this model, we find that $a g e_{\text {in }}<a g e_{\text {out }}$, consistent with the benchmark model; the fact that - by construction - $[\mathrm{Z} / \mathrm{H}]_{\text {in }}>[\mathrm{Z} / \mathrm{H}]_{\text {out }}$ yields a marginally higher $\chi_{\nu}^{2}=2.0$ compared to the benchmark $\chi_{\nu}^{2}=1.9$. The second model has free metallicity, but age $e_{\text {in }}>a g e_{\text {out }}$ (PSB insideout age model, Table 1). The best-fit parameters for this model predict flat $\mathrm{H} \delta_{\mathrm{A}}$ and $\mathrm{Fe} 4383$ radial profiles, inconsistent with observations. Quantitatively, the reduced $\chi_{\nu}^{2} \approx 6$ is larger than the value for the fiducial model $\left(\chi_{\nu}^{2}=1.9\right)$. We conclude that an inside-out age structure is inconsistent with the radial variations of $\mathrm{H} \delta_{\mathrm{A}}$ and $\mathrm{Fe} 4383$ observed for the stacked PSB galaxies. These two constrained models suggest that, while inside-out age gradients are ruled out for the PSB sample, both inside-out and inverse metallicity gradients are consistent with observations (cf. Cresci et al. 2010; Schönrich \& McMillan 2017).
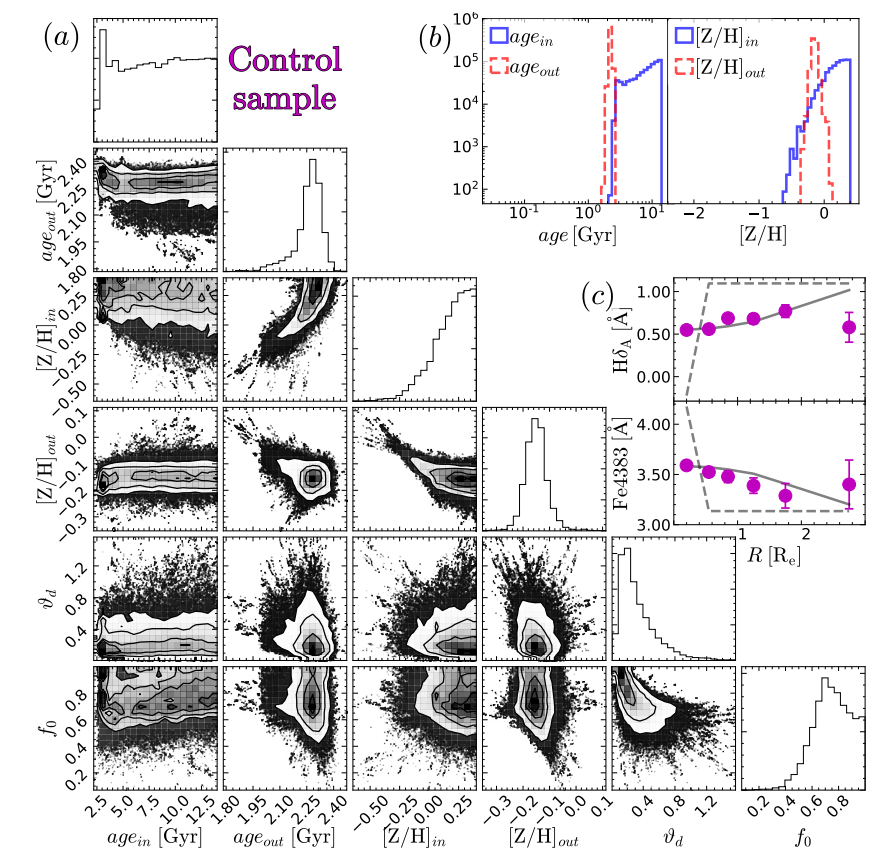

Figure 6. Our two-SSP toy model requires inside-out age and/or metallicity gradients for the control sample of quiescent, non PSB galaxies (panels a and b). The inferred model reproduces the median observed gradients in both $\mathrm{H} \delta_{\mathrm{A}}$ and Fe4383 (panel c; the dashed/solid lines show the model prediction before/after seeing convolution, and the magenta circles trace the median index values for our control sample). Even though the age of the inner SSP is poorly constrained, it is clear that age $e_{\text {in }}>$ age out (panel b): the inner SSP (solid blue histogram) is clearly older than the outer SSP (dashed red histogram, $P<3 \times 10^{-4}$ ). In contrast, even though $[\mathrm{Z} / \mathrm{H}]_{\text {in }}>[\mathrm{Z} / \mathrm{H}]_{\text {out }}$, the two SSP metallicities are consistent within the uncertainties $(P<0.07)$. Notice the strong degeneracy between the parameters of the two SSPs.

\subsection{Effect of extended star-formation history}

While an SSP is a good model for a starburst (where the spread in stellar age is narrow by definition), stellar populations are known to have extended star-formation histories (SFH). To what extent the different properties of SSPs and more realistic stellar population might bias our results? To address this question, we implemented a three-SSP model. For brevity, we refer to these SSPs by increasing roman numerals I-III. In order to preserve some predictive power, we want the smallest possible number of free parameters. For this reason, the three SSPs have different ages $\left(a g e_{\mathrm{I}}\right.$, $a g e_{\mathrm{II}}$ and $\left.a g e_{\mathrm{III}}\right)$ but equal metallicity $[\mathrm{Z} / \mathrm{H}]$. Even though this restriction is not realistic, it reflects the fact that, with our two indices, we do not find strong metallicity differences within the PSB sample (right panel of Fig. 4b). For the radial variation, we use the same parametrisation introduced for the benchmark model (Eq. 1; again, like we did for the benchmark model, we parametrise $R_{d}$ as $\tan \vartheta_{d}$, with $\left.0 \leq \vartheta_{d} \leq \pi / 2\right)$. Using the same parametrisation means that SSP I can be safely interpreted as the inner SSP from the benchmark model, whereas the outer SSP from the benchmark model corresponds here to the superposition of SSP II and SSP III: this superposition is the zero-order approximation for an extended SFH. At any radius $R$, the 
Table 1. Summary of the two-SSPs models. PSB galaxies are best described by an inverse stellar population structure, i.e. centre younger and/or lower metallicity than the outskirts. Imposing an inside-out age structure on PSB galaxies yields a poor fit (reduced $\left.\chi^{2} \approx 6\right)$. On the contrary, control galaxies are best described by an inside-out stellar population structure.

\begin{tabular}{|c|c|c|c|c|c|c|c|c|c|}
\hline $\begin{array}{c}\text { Model Name } \\
\text { (1) }\end{array}$ & $\begin{array}{c}\text { Constraints } \\
\text { (2) }\end{array}$ & $\begin{array}{c}\text { age }_{\text {in }} \\
\text { Gyr } \\
(3)\end{array}$ & $\begin{array}{l}\text { age }_{\text {out }} \\
\text { Gyr } \\
(4)\end{array}$ & $\begin{array}{c}{[\mathrm{Z} / \mathrm{H}]_{i n}} \\
(5)\end{array}$ & $\begin{array}{c}{[\mathrm{Z} / \mathrm{H}]_{\text {out }}} \\
(6)\end{array}$ & $\begin{array}{l}\vartheta_{d} \\
(7)\end{array}$ & $\begin{array}{l}f_{0} \\
(8)\end{array}$ & $\begin{array}{l}\chi_{\nu}^{2} \\
(9)\end{array}$ & $\begin{array}{l}\text { Structure } \\
\qquad(10)\end{array}$ \\
\hline $\begin{array}{c}\text { PSB } \\
\text { (benchmark) }\end{array}$ & none & $0.48_{-0.21}^{+0.23}$ & $1.28_{-0.20}^{+0.43}$ & $-0.13_{-0.22}^{+0.33}$ & $0.10_{-0.06}^{+0.17}$ & $1.41_{-0.29}^{+0.13}$ & $0.15_{-0.05}^{+0.11}$ & 1.9 & inverse \\
\hline $\begin{array}{c}\text { Control } \\
\text { (benchmark) }\end{array}$ & none & $8.3_{-4.2}^{+3.9}$ & $2.27_{-0.08}^{+0.05}$ & $0.21_{-0.19}^{+0.13}$ & $-0.15_{-0.04}^{+0.04}$ & $0.26_{-0.13}^{+0.28}$ & $0.75_{-0.14}^{+0.15}$ & 3.5 & inside-out \\
\hline $\begin{array}{l}\text { PSB inside-out } \\
\text { age }\end{array}$ & $a_{g} e_{\text {in }} \geq a g e_{\text {out }}$ & $3.95_{-3.10}^{+7.70}$ & $0.85_{-6.28}^{+0.02}$ & $0.08_{-0.69}^{+0.18}$ & $0.15_{-0.10}^{+0.07}$ & $1.20_{-0.87}^{+0.34}$ & $0.40_{-0.34}^{+0.59}$ & 6.0 & $\begin{array}{c}\text { inside-out (age) } \\
\text { inverse (metallicity) }\end{array}$ \\
\hline $\begin{array}{l}\text { PSB inside-out } \\
\text { metallicity }\end{array}$ & {$[\mathrm{Z} / \mathrm{H}]_{\text {in }} \geq[\mathrm{Z} / \mathrm{H}]_{\text {out }}$} & $0.68_{-0.11}^{+0.12}$ & $9.84_{-6.97}^{+2.99}$ & $0.18_{-0.08}^{+0.15}$ & $0.06_{-0.15}^{+0.13}$ & $1.51_{-0.08}^{+0.04}$ & $0.30_{-0.08}^{+0.14}$ & 2.0 & $\begin{array}{c}\text { inverse (age) } \\
\text { inside-out (metallicity) }\end{array}$ \\
\hline
\end{tabular}

(1) Name of the model as introduced in the main text. (2) Additional constraints on the age and metallicity of the two SSPs. (3) Inferred age of the central SSPs (here and in the following, we quote the median value of the marginalised posterior distribution; the uncertainties refer to the $16^{\text {th }}$ and $84^{\text {th }}$ percentile of the probability). (4) Inferred age of the outer SSP. (5) Inferred metallicity of the central SSP. (6) Inferred metallicity of the outer SSP. (7) Inferred value of the concentration parameter. (8) Inferred value of the mass fraction of the central SSP in the central pixel. (9) $\chi^{2}$ per degree of freedom. (10) Description of the model outcome: "inverse" refers to positive radial gradients in age and/or metallicity, "inside-out" refers to negative radial gradients in age and/or metallicity.

value of the mass fractions are $f_{\mathrm{I}}(R), f_{\mathrm{II}}(R)$ and $f_{\mathrm{III}}(R)$, that we parametrise using two variables $0 \leq \vartheta \leq \pi / 2$ and $0 \leq \varphi \leq \pi / 2$ :

$$
\begin{cases}f_{\mathrm{I}}(R) \equiv & \cos ^{2}(\vartheta) f(R) \\ f_{\mathrm{II}}(R) \equiv & {\left[1-\cos ^{2}(\vartheta) f(R)\right] \cos ^{2}(\varphi)} \\ f_{\mathrm{III}}(R) \equiv & {\left[1-\cos ^{2}(\vartheta) f(R)\right] \sin ^{2}(\varphi)}\end{cases}
$$

It can be easily verified that these functions express meaningful fractions, because they satisfy both $f_{\mathrm{I}}(R)+f_{\mathrm{II}}(R)+$ $f_{\mathrm{III}}(R)=1$ as well as $0 \leq f_{\mathrm{x}}(R) \leq 1 ; \forall x \in\{\mathrm{I}, \mathrm{II}, \mathrm{III}\}$. In particular, the angle $\vartheta$ expresses the fraction of SSP I at $R=0$, via $f_{\mathrm{I}}(0)=\cos ^{2}(\vartheta)$, whereas the angle $\varphi$ expresses the fraction, relative to the remaining stellar population, of SSP II (resp. SSP III) via $\cos ^{2}(\varphi)\left(\right.$ resp. $\left.\sin ^{2}(\varphi)\right)$. Notice that the fraction of SSP II decreases with increasing $\varphi$, whereas the fraction of SSP III increases with increasing $\varphi$. This model has an undesired symmetry, in that the results are unchanged swapping $\left(\operatorname{age}_{\mathrm{II}}, \operatorname{age}_{\mathrm{III}}, \varphi\right)$ with $\left(\right.$ age $\left._{\mathrm{III}}, a_{\mathrm{II}}, \pi / 2-\varphi\right)$, therefore we further require age $_{\mathrm{II}} \leq$ age $_{\text {III }}$ by assigning zero probability to non-complying models. This means that SSP II is always younger than SSP III, therefore the age of the outer SSP (equal to the sum of SSP II and SSP III) increases with increasing $\varphi$. So far the model has seven free parameters: age $e_{\mathrm{I}}, a g e_{\mathrm{II}}, a e_{\mathrm{III}}$, $[\mathrm{Z} / \mathrm{H}], \vartheta, \varphi$ and $\vartheta_{d}$. To reduce this number, we further constrain $\vartheta$ and $\vartheta_{d}$ to reproduce the corresponding optimal values from the benchmark model, i.e. we set $\vartheta_{d}=1.41$ and $\vartheta=\arccos \sqrt{f_{0}}$, with $f_{0}=0.15$ (first row of Table 1 ).

This model reproduces the observed index profiles as well as the benchmark model $\left(\chi_{\nu}^{2}=10^{1000}\right)$; the marginalised posterior probability is illustrated by the corner diagram in Fig. 7a. We find the usual age-metallicity degeneracy, between each SSP age and $[\mathrm{Z} / \mathrm{H}]$ (the Spearman rank correlation coefficients for $a g e_{\mathrm{I}}, a_{\mathrm{g}} \mathrm{II}$ and $a g e_{\mathrm{III}}$ are $\rho=-0.81, \rho=-0.21$ and $\rho=-0.10$ respectively). $\varphi$, the parameter governing the relative fraction of SSP II and SSP III, correlates with age $(\rho=0.30)$ : this positive cor- relation reflects the fact that the measured index profiles must be met by diluting a relatively younger/older starburst (SSP I) with a correspondingly younger/older outer SSP (SSP II + SSP III). This implies that our indices constrain only the mean age of the outer population. For this reason, $\varphi$ anti-correlates with age $e_{\mathrm{II}}(\rho=-0.16)$, because for a given value of $a g e_{\mathrm{I}}$ and $a g e_{\mathrm{III}}$, the required age of the outer SSP must be met either with a low fraction of young SSP II stars (larger $\varphi$ ) or with a high fraction of relatively older SSP II stars (lower $\varphi$ ). Similar reasoning explains the positive correlation between $\varphi$ and age $_{\mathrm{III}}$.

In Fig. 7b (left panel) we report the posterior distribution of the three SSPs: it can be seen that SSP II (dashed yellow histogram) overlaps with SSP III (dotted red histogram), but this does not mean that $a g e_{\mathrm{II}} \geq a g e_{\mathrm{III}}$, as can be seen from the joint probability distribution of age $\mathrm{II}$ and age $_{\mathrm{III}}$ (third row, second column of the corner diagram, Fig. 7a). It is instead true that SSP II is occasionally younger than SSP I (dashed yellow and solid blue histograms, respectively): this occurs with a probability $P<0.07$. However, even these $7 \%$ of cases do not contradict the main conclusion that PSB galaxies have inverse age gradients. In fact, what really matters is the mean age of the outer SSP, consisting of both SSP II and the older SSP III. The age of the outer SSP is illustrated in the right panel of Fig. 7b (dot-dashed red histogram), where we reproduce again the histogram of $a g e_{\mathrm{I}}$ for ease of comparison (solid blue line). In no case we find that the outer age is younger than the $a g e_{\mathrm{I}}$ (the overlap between the two histograms does not take into account the positive correlation between the age of the three SSPs). By comparing this histogram with the left panel of Fig. 5b, illustrating the results for the benchmark model, we see that the three-SSP model allows for much older outer SSPs than the benchmark model, reflecting the oversimplification of using a single SSP (in the benchmark model) instead of an extended star-formation history. Thus in general we can expect older ages for all of our non-starburst components. 


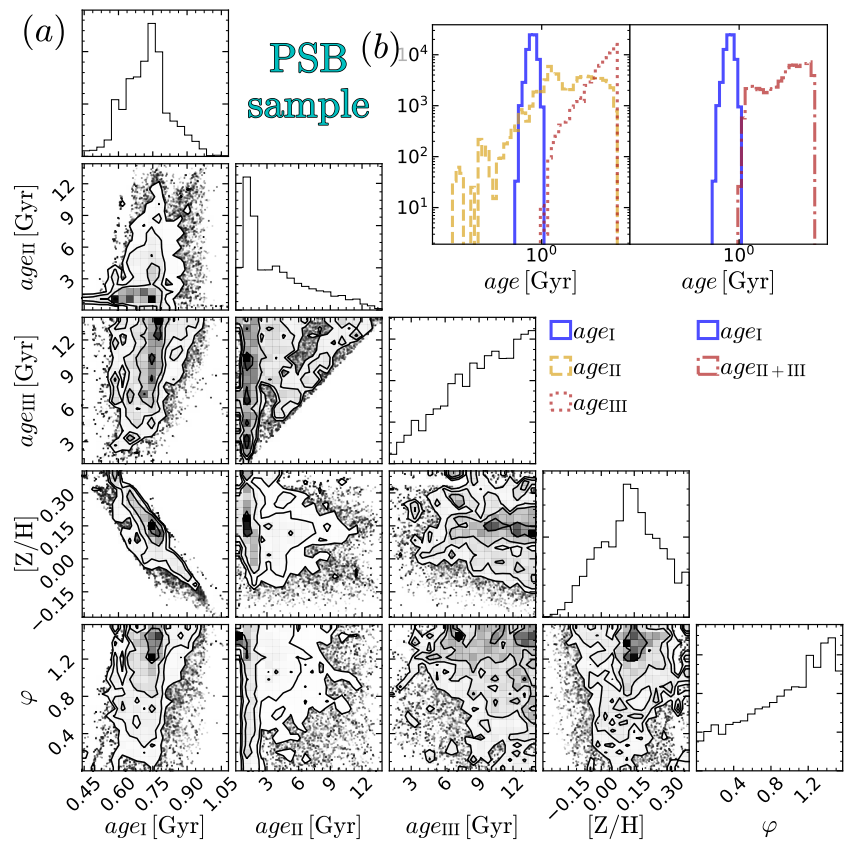

Figure 7. Assessing the effect of extended star-formation histories instead of SSPs for PSB galaxies. The model uses three SSPs: SSP I corresponds to the inner stellar population, whereas the superposition of the young SSP II and the old SSP III are a first-order approximation to an extended star-formation history. The corner diagram (panel a) shows that the fraction of SSP III stars $\left(\cos ^{2} \varphi\right)$ anti-correlates with age II (the age of the younger

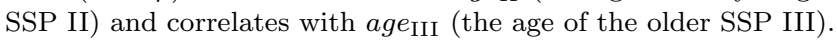
This behaviour reflects the fact that the indices considered here constrain only the average age of the outer stellar population: this value of the age can be attained with both a long or a short starformation history. Panel b, left, shows the probability distribution of the three SSP ages: $\operatorname{age}_{\mathrm{I}}$ (solid blue) is clearly younger than both age $_{\mathrm{II}}$ (dashed yellow) and age $\mathrm{III}_{\mathrm{II}}$ (dot-dashed red). Even though occasionally $a g e_{\mathrm{II}} \leq a g e_{\mathrm{I}}$, this occurs with relatively low probability $P<0.07$. More importantly, in the right panel we can see that the inner SSP is systematically younger than the outer SSP $P<10^{-5}$.

\section{DISCUSSION}

The different mass-size relations of SF and $\mathrm{Q}$ galaxies, as well as their evolution with cosmic time, require a link between star formation and structural evolution in galaxies (§1). There are several physical processes which can cause a SF galaxy to become quiescent, and each one of them might impart different structural signatures on newly-quiescent galaxies. For this reason, we can reasonably expect to learn something about how galaxies become quiescent by studying the structural differences between SF, Q and newlyquiescent galaxies. We can roughly divide quenching mechanisms in two classes, based on their timescale relative to the visibility time of PSB galaxies, strongly constrained by the lifetime of A-type stars ( $<1 \mathrm{Gyr})$.

Slow quenching processes act over a few Gyr, longer than the typical star-formation timescale at $z=0.8$ (defined as the typical inverse specific star-formation rate $s S F R^{-1} \approx$ 1 Gyr; Noeske et al. 2007). These processes include: (i) virial shocks (which prevent the accretion of cold gas, but leave the existing gas disc intact; Birnboim \& Dekel 2003; Dekel
\& Birnboim 2006), (ii) radio-mode feedback due to active galactic nuclei ${ }^{6}$ (AGN; Croton et al. 2006, Barišić et al. 2017) and (iii) stabilization of the gas disc against fragmentation ( $Q$-quenching; Martig et al. 2009; Cacciato et al. 2012; Forbes et al. 2014; Krumholz \& Thompson 2013; Dekel $\&$ Burkert 2014). These mechanisms cause little or no disruption to the gas that is currently fueling star formation, so that the galaxy can continue on the star-forming sequence for some time, until the cold-gas supply is either exhausted or otherwise unable to form stars. By definition, these mechanisms act gradually, thereby leaving a $\mathrm{Q}$ galaxy with roughly the same mass and size, and the same structure as the original SF galaxy. Most SF galaxies form in an inside-out fashion (Pezzulli et al. 2015; Ellison et al. 2018; Wang et al. 2019), leading to negative age gradients. At the same time, chemical enrichment models predict negative or flat stellar metallicity gradients (with some inversion in the centre, see Schönrich \& McMillan 2017). Thus the fact that our control sample of $\mathrm{Q}$ galaxies shows negative age and metallicity gradients ( $(4.2)$ is qualitatively consistent with the slow quenching and subsequent passive evolution of SF galaxies from earlier epochs, without major structural changes. There are two important caveats to this conclusion. Firstly, our results are derived from stacks, so they are valid only in an average sense: we cannot say whether (or what fraction of) passive galaxies had more complex starformation histories, with star-formation ending last in the centre, or with later central starbursts (rejuvenation; see Chauke et al. 2018, for an integrated analysis using LEGAC). Secondly, and more importantly, we remark that control galaxies became quiescent at earlier epochs compared to $z \approx 0.8$ PSB galaxies, at a time when the star-formation timescale was shorter.

On the other hand, fast quenching processes happen on relatively short timescales $(\lesssim 1 \mathrm{Gyr}$, e.g. Kaviraj et al. 2007; Dekel \& Burkert 2014), shorter than the timescale of star formation. These processes involve, in one way or another, the removal of the currently star-forming gas: through AGN-driven galactic-scale winds (Springel et al. 2005; Kaviraj et al. 2007; Baron et al. 2018), through ram-pressure stripping (in galaxy clusters; Gunn \& Gott $1972)^{7}$, or via rapid gas-consumption in gas-rich mergers (Barnes \& Hernquist 1991, 1996; Hopkins et al. 2009; Dekel \& Burkert 2014). A key property of the fast quenching processes is that all of them either require or cause the presence of centrally-concentrated cold gas. This gas builds up a dense stellar core, thereby increasing the stellar mass and shrinking the half-light radius of the underlying galaxy just before it becomes quiescent. Some of the fast-quenching mechanisms also produce an increasing age trend with radius (Mihos \& Hernquist 1994; Bekki et al. 2005), a signature opposite to the negative age gradients expected from inside-out formation.

6 but notice that, depending on the angle of the radio jet with respect to the gas disc, radio-mode feedback may lead to molecular outflows, see e.g. García-Burillo et al. 2014; Sakamoto et al. 2014; Morganti et al. 2015; Dasyra et al. 2016

7 Given that our sample consists mostly of central/isolated galaxies, we ignore ram-pressure stripping from here on. 


\subsection{Evidence of central starbursts in PSB galaxies}

Post-starburst galaxies are passive galaxies with a significant fraction of young stars. They lack current star formation on timescales of 10-100 Myr (depending on the tracer used) and they have prominent Balmer spectral features typical of A-type stars, which have lifetimes of $<1$ Gyr. These two timescales constrain the quenching timescale of PSB galaxies, placing them decisively in the fast-quenching channel. We argue that the structural properties of PSB galaxies are in qualitative agreement with the expectations of the fastquenching scenario described above.

Firstly, our PSB galaxies show a high merger fraction (0.40) relative to field galaxies $(0.16 \pm 0.02$ at $z \approx 1$ Mantha et al. 2018, their Table 2). Given our sample size and assuming Poisson uncertainties, the probability that the PSB sample has the same merger rate as observed in the field is relatively modest, $P=0.02$. This suggests that gas-rich mergers are an important trigger of PSB evolution, in agreement with the theoretical scenario proposed above. Notice, however, that the value $P=0.02$ assumes that the merger classification was perfect. In practice, there are two main uncertainties that are hard to quantify in our small sample: misclassification can bias the $P$-value to lower values (e.g. asymmetries due to dust and chance alignments misclassfied as mergers), whereas uncertainties on the visibility time of merger signatures bias the $P$-value to higher values. Nevertheless, our results are qualitatively unchanged if we repeat the test separately for merging and non-merging PSBs (§3), therefore it is unlikely that the high-visibility mergers we are able to discern are the only channel for PSB formation: the remaining $60 \%$ of PSB galaxies that show no evidence of major mergers could either: i) be mergers below our detection threshold (e.g. Zabludoff et al. 1996, Goto 2005, Yang et al. 2008, Pracy et al. 2009 find merger fractions between 15 and 70 per cent, depending on sample selection and image quality, see Pawlik et al. 2018 for a discussion), or ii) have accreted low-stellar-mass, gas-rich satellites, or iii) have undergone a qualitatively different mode of gas accretion, such as cold flows (Dekel et al. 2009). Regardless, all of these channels are consistent with the fast quenching scenario. This high but not overwhelming merger fraction is consistent with the prediction of cosmological Nbody simulations, which suggest roughly half of PSB galaxies did not experience a recent merger (Wilkinson et al. 2018; Davis et al. 2019; Pawlik et al. 2019).

Secondly, stacking analysis of $z>1$ PSB galaxies show evidence of high-velocity outflows (Maltby et al. 2019), capable of removing the star-forming gas and thus halting star formation abruptly. There is at least one case where this process has been observed in act (Baron et al. 2018). These outflows may temporarily delay star formation, until other physical processes, acting on longer timescales, can permanently halt the supply of cold gas and make the galaxy quiescent.

Moreover, the structural changes expected from fast quenching go in the right direction vis-à-vis the observed differences between normal and PSB galaxies on the masssize plane: at a given stellar mass, PSB galaxies are slightly smaller than normal Q galaxies and are much smaller than SF galaxies (Whitaker et al. 2012; Yano et al. 2016; Wu et al. 2018).
Finally, in this work we provide direct evidence that PSB galaxies at $z \approx 0.8$ have younger stellar populations in their centres, thereby demonstrating that, in these galaxies, star formation occurred last in the centre. This event is different from the typical inside-out formation that characterises both the control sample (Fig. 4, magenta) as well as the bulk of local SF galaxies (e.g. González Delgado et al. 2015; Zibetti et al. 2017; Ellison et al. 2018).

\subsection{The progenitors of PSB galaxies}

The discovery that PSB galaxies have inverse stellar population gradients is in agreement with the hypothesis that PSB galaxies are descendants of compact star-forming (CSF) galaxies. These galaxies are characterised by high starformation rates and small size relative to $\mathrm{SF}$ galaxies of the same mass (star-formation rate $>100 \mathrm{M}_{\odot} \mathrm{yr}^{-1}, R_{e}<3 \mathrm{kpc}$; e.g. Barro et al. 2013, 2014; van Dokkum et al. 2015). Yet this high star-formation rate is mostly undetected in the rest-frame optical wavelengths because it is obscured by large optical depth.

Even though the number density of CSF galaxies is too high compared to that of PSB galaxies (Wild et al. 2016), CSF galaxies are not guaranteed to become quiescent, hence their number density does not need to match exactly that of PSB galaxies. For example, CSF galaxies could transition to a state of lowered star-formation rate instead of becoming quiescent.

On the other hand, there is evidence that CSF galaxies, too, have inverse stellar population gradients, because their star formation is less extended than the already-formed stellar population (Barro et al. 2016, 2017; Tadaki et al. 2017; Popping et al. 2017). If CSF galaxies transitioned to a relatively long phase of lowered star-formation rate, characterised by inside-out growth, the inverse signature on their stellar populations might be erased (at least in part, giving rise to U-shaped age profiles observed in some local early-type galaxies Zibetti et al. 2020). If at least some of these galaxies were about to undergo a rapid cessation of star formation, the inverse gradients would be "frozen". Our finding that PSB galaxies harbour inverse stellar population gradients agrees with the hypothesis that at least some of their progenitors might be CSF galaxies that underwent rapid quenching.

Alternatively, the progenitors of PSB galaxies could be galaxies detected at sub-mm wavelength (sub-mm galaxies Smail et al. 1997; Hughes et al. 1998; Barger et al. 1998; Eales et al. 1999). These galaxies are characterised by high star-formation rates (Swinbank et al. 2014), consistent with the star-formation history of PSB galaxies (Wild et al. 2020). Moreover, the combination of the number density of sub-mm galaxies $(\approx 6$ times higher than that of PSB Swinbank et al. 2014; Simpson et al. 2014) and the visibility time of the submm phase $(\approx 6$ times shorter than that of PSB Hainline et al. 2011; Hickox et al. 2012) cancel out and match the observed number density of observed PSB galaxies (Wild et al. 2020). 


\subsection{Caveats}

Given our sample selection criteria, it is difficult to assess the generality of our results vis-á-vis the unbiased population of PSB galaxies at redshift $z \approx 0.8$. In fact, our SNR and spatial resolution bias the sample towards the largest and brightest PSB galaxies, therefore a trend between stellarpopulation gradients and size or luminosity could mean that our conclusions are not representative of the whole PSB population. Conversely, removing galaxies with small $R_{e}$ means that our sample is biased against the most compact PSB galaxies, which may represent the youngest galaxies in this category (Wu et al. 2020). For these reasons, we cannot draw stringent conclusions concerning how size affects the properties of our galaxies (but we remark that jackknifing our sample about the median $R_{\mathrm{e}}$ yields consistent results for the smallest and largest PSB considered here). However, we can still robustly conclude that inverse age gradients are representative of $\approx 50 \%$ of the PSB population (even though individual PSB galaxies can indeed have inside-out gradients, e.g. 258467 has a positive $\mathrm{H} \delta_{\mathrm{A}}$ gradient with four standard deviations; see also Appendix B and Hunt et al. 2018). Moreover, our data suggest that, provided the galaxies are at least partially resolved, there is no strong dependence of the incidence of inverse gradients with either galaxy size or galaxy concentration. Finally, we find that our results cannot be explained by environment effects (i.e. rampressure stripping), because the inverse gradients persist for central/isolated galaxies only.

Using the UVJ diagram to select Q galaxies may bias our sample, by including misclassified SF galaxies. There are two indications that this is not the case. Firstly, if we select only PSB galaxies where the wavelength range includes restframe $\mathrm{H} \beta$, and if for these galaxies we further require equivalent width $E W(\mathrm{H} \beta) \geq 1 \AA$, we still find inverse gradients for $\mathrm{H} \delta_{\mathrm{A}}$ (emission lines have negative EW). For Fe4383 the gradients are consistent with being flat, but this is likely due to the smaller sample size. Secondly, a preliminary analysis of SF galaxies finds inside-out gradients, similar to non-PSB quiescent galaxies, therefore any contamination would make our results weaker.

Another caveat is that, at least for PSB galaxies, the SNR of $\mathrm{H} \delta_{\mathrm{A}}$ is higher than the SNR of Fe4383, which might skew our analysis. Furthermore, we are unable to constrain the abundance of $\alpha$-elements relative to Fe, which is important to properly constrain the metallicity of a stellar population (e.g. González 1993; Thomas et al. 2003; Conroy 2013), We note though that this should not be a concern for determining the age of the PSB galaxies (Leonardi \& Rose 1996), but might affect the older control sample.

Unfortunately at present we cannot derive strong quantitative constraints on the stellar population gradients in PSB galaxies: seeing convolution prevents us from breaking the degeneracy between spatial distribution, age and mass fraction of the young, central sub-population. Still, the observed agreement with local observations is promising ( $\S 4.1)$. In addition, the need for stacking data from different galaxies means that our models do not necessarily capture the unbiased average of the population. Individual galaxies show a plethora of radial behaviours, as suggested by resolved studies of individual galaxies in the local Universe (e.g. Pracy et al. 2013; Owers et al. 2019; Chen et al.
2019): the fact that some PSB galaxies have flat gradients is not in contradiction with our results. These different radial behaviours may depend on the timing and on the properties of the burst.

\subsection{Future work}

Current observing facilities are unable to disentangle this degeneracy, therefore we foresee a multi-pronged approach.

Space-based observations have enough spatial resolution to resolve the inner structure of PSB galaxies. Unfortunately, at the time of this writing, the number of PSB galaxies with adequate photometry in two bands is limited, and cannot be used to draw strong conclusions for our sample (Appendix B). At variance with our findings, Maltby et al. (2018) find no evidence of colour gradients in a sample of 80 PSB galaxies at $0.5<z<2$, but they rely on photometry only, therefore a direct comparison is not warranted.

Another implication of the scenario proposed is that the youngest PSB galaxies ought to have the smallest half-light radii. This is because the younger central population has a lower mass-to-light ratio, but dims faster than the older, extended population (as indeed confirmed by Wu et al. 2020; see their discussion for the effect of dust).

Studying the structure of local galaxies is complicated by the intervening evolution between $z \approx 0.8$ and now, but inverse age structures have been found in the most massive early-type galaxies (Zibetti et al. 2020). This approach should be combined with a study of star-burst dwarf galaxies in the local Universe. These systems have many properties in common with higher-mass galaxies in the early Universe (e.g. Lelli et al. 2014; Yang et al. 2017). Intriguingly, some of these local galaxies have inverse age structures caught in formation (Zhang et al. 2012), and might shed light on the physical processes occurring at high gas fractions.

Accurate modelling involving more than indices (e.g. Zibetti et al. 2017) may also provide more stringent constraints on the age and metallicity of PSB galaxies, resolving some of the degeneracies inherent to using only two indices (\$4.1). The upcoming third data release of LEGA-C will likely improve both the precision and the accuracy of our measurements. Moreover, owing to a larger sample and thanks to the improved sky subtraction at the reddest wavelengths, it will unlock additional stellar-population information.

\section{SUMMARY}

We have used a sample of seventeen UVJ-colour selected, spectrally-confirmed post-starburst (PSB) galaxies from the deep, $z \approx 0.8$ LEGA-C Survey, to study the radial variation of the strength of the $\mathrm{H} \delta_{\mathrm{A}}$ and $\mathrm{Fe} 4383$ absorption indices. We find that:

- PSB galaxies show negative $\mathrm{H} \delta_{\mathrm{A}}$ radial gradients, and positive Fe4383 gradients ( $P$-values $10^{-4}$ and 0.04 respectively).

- the control sample of quiescent, non-PSB galaxies, selected to match the mass range of the PSB sample, presents positive $\mathrm{H} \delta_{\mathrm{A}}$ radial gradients and negative $\mathrm{Fe} 4383$ radial gradients ( $P$-values 0.07 and 0.02 respectively) 
- these trends imply a positive (inverse) age gradient with radius for PSB galaxies, and a negative age and/or metallicity gradient for control quiescent galaxies (the control sample).

- coupled with the mass and size distribution of PSB and quiescent galaxies, and with the higher-than-average merger fraction (0.4), our data suggest that PSB galaxies have undergone a central starburst, which decreased their effective radius and possibly caused them to become quiescent on a fast timescale ( $<1 \mathrm{Gyr})$.

\section{ACKNOWLEDGMENTS}

We thank the anonymous referee for helpful comments and suggestions. Based on observations made with ESO Telescopes at the La Silla Paranal Observatory under programme ID 194-A.2005 (The LEGA-C Public Spectroscopy Survey). This project has received funding from the European Research Council (ERC) under the European Union's Horizon 2020 research and innovation programme (grant agreement No. 683184). We are grateful to N. Scott for sharing the spectral fitting code. PFW acknowledges the support of the East Asian Core Observatories Association fellowship. TMB is supported by an Australian Government Research Training Program Scholarship. AG and SZ acknowledge support from Istituto Nazionale di Astrofisica (PRIN-SKA2017 program 1.05.01.88.04)

This work made extensive use of the Debian GNU/Linux operative system, freely available at http://www.debian.org. We used the Python programming language (van Rossum 1995), maintained and distributed by the Python Software Foundation, and freely available at http://www.python.org. We acknowledge the use of SCIPY (Jones et al. 2001), MATPLOTLIB (Hunter 2007), EMCEE (Foreman-Mackey et al. 2013), ASTROPY (Astropy Collaboration et al. 2013) and PATHOS (McKerns et al. 2011). During the preliminary analysis we have made extensive use of TOPCAT (Taylor 2005).

\section{DATA AVAILABILITY}

The raw data used in this work is available in the public domain, through the ESO Science Archive and through the Mikulski Archive for Space Telescopes (MAST). Part of the reduced data has been released with the second public data release of LEGA-C (Straatman et al. 2018), available on the LEGA-C website. Resolved measurements will be available in the upcoming third public data release of LEGA-C.

\section{References}

Almaini O., et al., 2017, MNRAS, 472, 1401

Astropy Collaboration et al., 2013, A\&A, 558, A33

Balogh M. L., Morris S. L., Yee H. K. C., Carlberg R. G., Ellingson E., 1997, ApJ, 488, L75

Balogh M. L., Morris S. L., Yee H. K. C., Carlberg R. G., Ellingson E., 1999, ApJ, 527, 54

Barger A. J., Cowie L. L., Sanders D. B., Fulton E., Taniguchi Y., Sato Y., Kawara K., Okuda H., 1998, Nature, 394, 248

Barišić I., et al., 2017, ApJ, 847, 72
Barnes J. E., Hernquist L. E., 1991, ApJ, 370, L65

Barnes J. E., Hernquist L., 1996, ApJ, 471, 115

Baron D., et al., 2018, MNRAS, 480, 3993

Barone T. M., D'Eugenio F., Colless M., Scott N., 2020, arXiv e-prints, p. arXiv:2006.00720

Barro G., et al., 2013, ApJ, 765, 104

Barro G., et al., 2014, ApJ, 791, 52

Barro G., et al., 2016, ApJ, 827, L32

Barro G., et al., 2017, ApJ, 851, L40

Bekki K., Shioya Y., Couch W. J., 2001, ApJ, 547, L17

Bekki K., Couch W. J., Shioya Y., Vazdekis A., 2005, MNRAS, 359, 949

Belli S., Newman A. B., Ellis R. S., 2017, ApJ, 834, 18

Bertelli G., Nasi E., Girardi L., Marigo P., 2009, A\&A, 508, 355

Bezanson R., et al., 2018, ApJ, 858, 60

Birnboim Y., Dekel A., 2003, MNRAS, 345, 349

Buck T., Obreja A., Macciò A. V., Minchev I., Dutton A. A., Ostriker J. P., 2019, arXiv e-prints, p. arXiv:1909.05864

Cacciato M., Dekel A., Genel S., 2012, MNRAS, 421, 818

Cappellari M., Emsellem E., 2004, PASP, 116, 138

Cappellari M., et al., 2013, MNRAS, 432, 1709

Carlberg R. G., Morris S. L., Yee H. K. C., Ellingson E., 1997, ApJ, 479, L19

Chabrier G., 2003, PASP, 115, 763

Chauke P., et al., 2018, ApJ, 861, 13

Chen Y.-M., et al., 2019, MNRAS, 489, 5709

Conroy C., 2013, ARA\&A, 51, 393

Couch W. J., Sharples R. M., 1987, MNRAS, 229, 423

Cresci G., Mannucci F., Maiolino R., Marconi A., Gnerucci A., Magrini L., 2010, Nature, 467, 811

Croton D. J., et al., 2006, MNRAS, 365, 11

Darvish B., Mobasher B., Martin D. C., Sobral D., Scoville N., Stroe A., Hemmati S., Kartaltepe J., 2017, ApJ, 837, 16

Dasyra K. M., Combes F., Oosterloo T., Oonk J. B. R., Morganti R., Salomé P., Vlahakis N., 2016, A\&A, 595, L7

Davis T. A., van de Voort F., Rowlands K., McAlpine S., Wild V., Crain R. A., 2019, MNRAS, 484, 2447

Dekel A., Birnboim Y., 2006, MNRAS, 368, 2

Dekel A., Burkert A., 2014, MNRAS, 438, 1870

Dekel A., et al., 2009, Nature, 457, 451

Dressler A., Gunn J. E., 1983, ApJ, 270, 7

Dressler A., Smail I., Poggianti B. M., Butcher H., Couch W. J., Ellis R. S., Oemler Augustus J., 1999, ApJS, 122, 51

Eales S., Lilly S., Gear W., Dunne L., Bond J. R., Hammer F., Le Fèvre O., Crampton D., 1999, ApJ, 515, 518

Ellison S. L., Sánchez S. F., Ibarra-Medel H., Antonio B., Mendel J. T., Barrera-Ballesteros J., 2018, MNRAS, 474, 2039

Fagioli M., Carollo C. M., Renzini A., Lilly S. J., Onodera M., Tacchella S., 2016, ApJ, 831, 173

Falcón-Barroso J., Sánchez-Blázquez P., Vazdekis A., Ricciardelli E., Cardiel N., Cenarro A. J., Gorgas J., Peletier R. F., 2011, A\&A, 532, A95

Ferreras I., et al., 2019, MNRAS, p. 2057

Forbes J. C., Krumholz M. R., Burkert A., Dekel A., 2014, MNRAS, 438, 1552

Foreman-Mackey D., Hogg D. W., Lang D., Goodman J., 2013, PASP, 125, 306

García-Burillo S., et al., 2014, A\&A, 567, A125

González J. J., 1993, PhD thesis, -

González Delgado R. M., et al., 2015, A\&A, 581, A103

Goto T., 2005, MNRAS, 357, 937

Grogin N. A., et al., 2011, ApJS, 197, 35

Gunn J. E., Gott III J. R., 1972, ApJ, 176, 1

Hainline L. J., Blain A. W., Smail I., Alexand er D. M., Armus L., Chapman S. C., Ivison R. J., 2011, ApJ, 740, 96

Hickox R. C., et al., 2012, MNRAS, 421, 284

Hiner K. D., Canalizo G., 2015, ApJ, 799, 59 
Hopkins P. F., Cox T. J., Dutta S. N., Hernquist L., Kormendy J., Lauer T. R., 2009, ApJS, 181, 135

Hughes D. H., et al., 1998, Nature, 394, 241

Hunt Q., et al., 2018, ApJ, 860, L18

Hunter J. D., 2007, Computing In Science \& Engineering, 9, 90

Jones E., Oliphant T., Peterson P., et al., 2001, SciPy: Open source scientific tools for Python, http://www.scipy.org/

Kauffmann G., et al., 2003, MNRAS, 341, 33

Kaviraj S., Kirkby L. A., Silk J., Sarzi M., 2007, MNRAS, 382, 960

Koekemoer A. M., et al., 2007, ApJS, 172, 196

Krumholz M. R., Thompson T. A., 2013, MNRAS, 434, 2329

La Barbera F., Ferreras I., de Carvalho R. R., Bruzual G., Charlot S., Pasquali A., Merlin E., 2012, MNRAS, 426, 2300

Labbé I., et al., 2005, ApJ, 624, L81

Le Fèvre O., et al., 2003, in Iye M., Moorwood A. F. M., eds, Proc. SPIEVol. 4841, Instrument Design and Performance for Optical/Infrared Ground-based Telescopes. pp 1670-1681, doi:10.1117/12.460959

Lelli F., Verheijen M., Fraternali F., 2014, A\&A, 566, A71

Leonardi A. J., Rose J. A., 1996, AJ, 111, 182

Maltby D. T., Almaini O., Wild V., Hatch N. A., Hartley W. G., Simpson C., Rowlands K., Socolovsky M., 2018, MNRAS, 480, 381

Maltby D. T., et al., 2019, MNRAS, 489, 1139

Mantha K. B., et al., 2018, MNRAS, 475, 1549

Martig M., Bournaud F., Teyssier R., Dekel A., 2009, ApJ, 707, 250

Martig M., Minchev I., Ness M., Fouesneau M., Rix H.-W., 2016, ApJ, 831, 139

Martín-Navarro I., Vazdekis A., Falcón-Barroso J., La Barbera F., Yıldırım A., van de Ven G., 2018, MNRAS, 475, 3700

Martins L. P., Coelho P., 2007, MNRAS, 381, 1329

McDermid R. M., et al., 2015, MNRAS, 448, 3484

McKerns M. M., Strand L., Sullivan T., Fang A., Aivazis M. A. G., 2011, in Proceedings of the 10th Python in Science Conference. (arXiv:1202.1056)

Metropolis N., Rosenbluth A. W., Rosenbluth M. N., Teller A. H., Teller E., 1953, The Journal of Chemical Physics, 21, 1087

Mihos J. C., Hernquist L., 1994, ApJ, 427, 112

Morganti R., Oosterloo T., Oonk J. B. R., Frieswijk W., Tadhunter C., 2015, A\&A, 580, A1

Mowla L. A., et al., 2019, ApJ, 880, 57

Muzzin A., et al., 2012, ApJ, 746, 188

Muzzin A., et al., 2013, ApJS, 206, 8

Nelson E. J., et al., 2016, ApJ, 828, 27

Noeske K. G., et al., 2007, ApJ, 660, L43

Norton S. A., Gebhardt K., Zabludoff A. I., Zaritsky D., 2001, ApJ, 557, 150

Oke J. B., Gunn J. E., 1983, ApJ, 266, 713

Owers M. S., et al., 2019, ApJ, 873, 52

Paccagnella A., Vulcani B., Poggianti B. M., Moretti A., Fritz J., Gullieuszik M., Fasano G., 2019, MNRAS, 482, 881

Paulino-Afonso A., Sobral D., Buitrago F., Afonso J., 2017, MNRAS, 465, 2717

Pawlik M. M., et al., 2018, MNRAS, 477, 1708

Pawlik M. M., McAlpine S., Trayford J. W., Wild V., Bower R., Crain R. A., Schaller M., Schaye J., 2019, Nature Astronomy, 3,440

Peng C. Y., Ho L. C., Impey C. D., Rix H.-W., 2010, AJ, 139, 2097

Pezzulli G., Fraternali F., Boissier S., Muñoz-Mateos J. C., 2015, MNRAS, 451, 2324

Pietrinferni A., Cassisi S., Salaris M., Castelli F., 2004, ApJ, 612, 168

Pietrinferni A., Cassisi S., Salaris M., Castelli F., 2006, ApJ, 642, 797
Plez B., 2011, in Journal of Physics Conference Series. p. 012005, doi:10.1088/1742-6596/328/1/012005

Poci A., McDermid R. M., Zhu L., van de Ven G., 2019, MNRAS, 487, 3776

Poggianti B. M., Smail I., Dressler A., Couch W. J., Barger A. J., Butcher H., Ellis R. S., Oemler Augustus J., 1999, ApJ, 518, 576

Poggianti B. M., et al., 2009, ApJ, 693, 112

Popping G., et al., 2017, A\&A, 602, A11

Pracy M. B., Kuntschner H., Couch W. J., Blake C., Bekki K., Briggs F., 2009, MNRAS, 396, 1349

Pracy M. B., et al., 2013, MNRAS, 432, 3131

Prugniel P., Soubiran C., 2001, A\&A, 369, 1048

Rousseeuw P. J., Driessen K., 2006, Data Min. Knowl. Discov., 12,29

Rowlands K., et al., 2018, MNRAS, 473, 1168

Sakamoto K., Aalto S., Combes F., Evans A., Peck A., 2014, ApJ, 797,90

Sánchez-Blázquez P., et al., 2006, MNRAS, 371, 703

Schönrich R., McMillan P. J., 2017, MNRAS, 467, 1154

Scott N., et al., 2017, MNRAS, 0, 0

Scoville N., et al., 2007, ApJS, 172, 38

Serra P., Trager S. C., 2007, MNRAS, 374, 769

Shen S., Mo H. J., White S. D. M., Blanton M. R., Kauffmann G., Voges W., Brinkmann J., Csabai I., 2003, MNRAS, 343, 978

Simpson J. M., et al., 2014, ApJ, 788, 125

Smail I., Ivison R. J., Blain A. W., 1997, ApJ, 490, L5

Springel V., Di Matteo T., Hernquist L., 2005, ApJ, 620, L79

Straatman C. M. S., et al., 2018, ApJS, 239, 27

Suzuki T. L., Minowa Y., Koyama Y., Kodama T., Hayashi M., Shimakawa R., Tanaka I., Tadaki K.-i., 2019, PASJ, 71, 69

Swinbank A. M., et al., 2014, MNRAS, 438, 1267

Tadaki K.-i., et al., 2017, ApJ, 841, L25

Taylor M. B., 2005, in Shopbell P., Britton M., Ebert R., eds, Astronomical Society of the Pacific Conference Series Vol. 347, Astronomical Data Analysis Software and Systems XIV. p. 29

Thomas D., Maraston C., Bender R., 2003, MNRAS, 339, 897

Trager S. C., Worthey G., Faber S. M., Burstein D., González J. J., 1998, ApJS, 116, 1

Tran K.-V. H., Franx M., Illingworth G. D., van Dokkum P., Kelson D. D., Magee D., 2004, ApJ, 609, 683

Valenti E., Zoccali M., Renzini A., Brown T. M., Gonzalez O. A., Minniti D., Debattista V. P., Mayer L., 2013, A\&A, 559, A98

Vazdekis A., Sánchez-Blázquez P., Falcón-Barroso J., Cenarro A. J., Beasley M. A., Cardiel N., Gorgas J., Peletier R. F., 2010, MNRAS, 404, 1639

Vazdekis A., et al., 2015, MNRAS, 449, 1177

Wang E., Lilly S. J., Pezzulli G., Matthee J., 2019, ApJ, 877, 132

Whitaker K. E., Kriek M., van Dokkum P. G., Bezanson R., Brammer G., Franx M., Labbé I., 2012, ApJ, 745, 179

Wild V., Walcher C. J., Johansson P. H., Tresse L., Charlot S., Pollo A., Le Fèvre O., de Ravel L., 2009, MNRAS, 395, 144

Wild V., Almaini O., Dunlop J., Simpson C., Rowlands K., Bowler R., Maltby D., McLure R., 2016, MNRAS, 463, 832

Wild V., et al., 2020, arXiv e-prints, p. arXiv:2001.09154

Wilkinson C. L., Pimbblet K. A., Stott J. P., Few C. G., Gibson B. K., 2018, MNRAS, 479, 758

Williams C. C., et al., 2017, ApJ, 838, 94

Worthey G., 1994, ApJS, 95, 107

Worthey G., Ottaviani D. L., 1997, ApJS, 111, 377

Wu P.-F., et al., 2018, ApJ, 868, 37

Wu P.-F., et al., 2020, ApJ, 888, 77

Yang Y., Zabludoff A. I., Zaritsky D., Lauer T. R., Mihos J. C., 2004, ApJ, 607, 258

Yang Y., Zabludoff A. I., Zaritsky D., Mihos J. C., 2008, ApJ, 688,945

Yang H., Malhotra S., Rhoads J. E., Wang J., 2017, ApJ, 847, 38 
Yano M., Kriek M., van der Wel A., Whitaker K. E., 2016, ApJ, 817, L21

Younger J. D., Bahcall N. A., Bode P., 2005, ApJ, 622, 1

Zabludoff A. I., Zaritsky D., Lin H., Tucker D., Hashimoto Y., Shectman S. A., Oemler A., Kirshner R. P., 1996, ApJ, 466, 104

Zhang H.-X., Hunter D. A., Elmegreen B. G., Gao Y., Schruba A., 2012, AJ, 143, 47

Zibetti S., et al., 2017, MNRAS, 468, 1902

Zibetti S., Gallazzi A. R., Hirschmann M., Consolandi G., FalcónBarroso J., van de Ven G., Lyubenova M., 2020, MNRAS, 491, 3562

van Dokkum P. G., et al., 2015, ApJ, 813, 23

van Rossum G., 1995, Technical Report, CS-R9526

van de Sande J., et al., 2017, ApJ, 835, 104

van der Wel A., Bell E. F., van den Bosch F. C., Gallazzi A., Rix H.-W., 2009, ApJ, 698, 1232

van der Wel A., et al., 2012, ApJS, 203, 24

van der Wel A., et al., 2014, ApJ, 788, 28

van der Wel A., et al., 2016, ApJS, 223, 29

\section{APPENDIX A: $\mathrm{H} \gamma_{\mathrm{A}}$ AND $\mathrm{D}_{\mathrm{n}} 4000$ INDICES}

The PSB and control sample have qualitatively different radial trends also in their $\mathrm{D}_{\mathrm{n}} 4000$ and $\mathrm{H} \gamma_{\mathrm{A}}$ indices (Fig. A1)

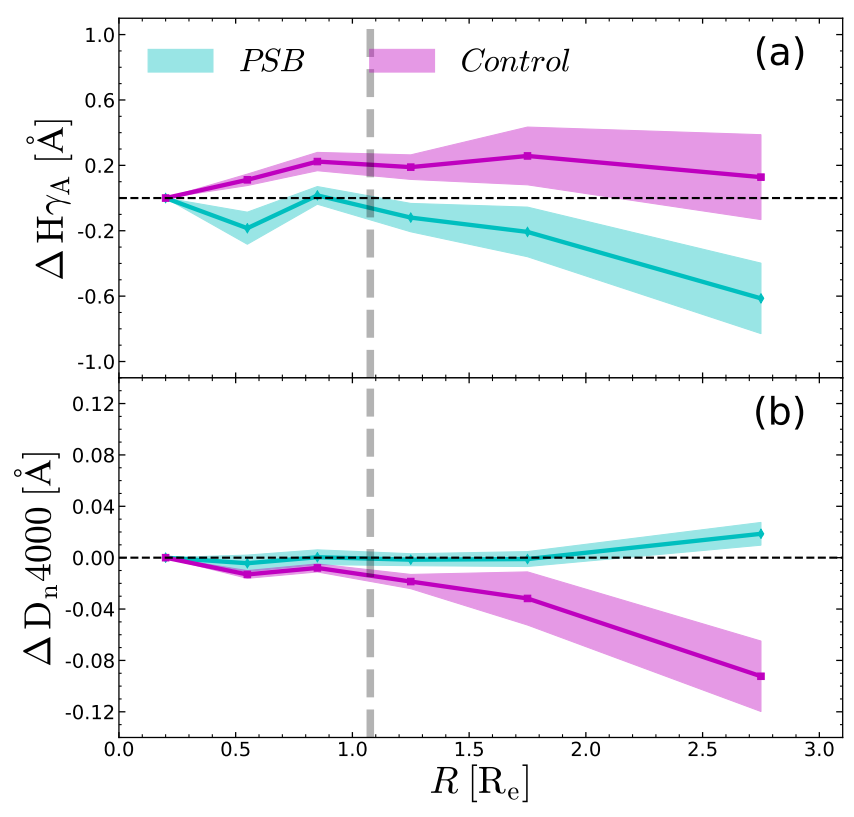

Figure A1. Same as Fig. 4, but for $\mathrm{H} \gamma_{\mathrm{A}}$ (panel a), and $\mathrm{D}_{\mathrm{n}} 4000$ (panel b). These two indices show again that PSB galaxies (cyan) have different radial profiles than the control sample of quiescent, non-PSB galaxies (magenta). For PSB galaxies, $\mathrm{H} \gamma_{\mathrm{A}}$ is radially decreasing and $D_{n} 4000$ is flat, whereas for control galaxies, $\mathrm{H} \gamma_{A}$ is radially increasing and $\mathrm{D}_{\mathrm{n}} 4000$ is radially decreasing.

\section{APPENDIX B: COLOUR GRADIENTS}

Depending on the strength of the intrinsic age gradient, the presence of inverse stellar gradients in PSB galaxies can be investigated using space-based photometry. For a meaningful test, we require two conditions: firstly, we need spacebased photometry to derive intrinsic radial profiles of restframe optical colour. Secondly, we need to compare these radial colour profiles to the index profiles of the relevant galaxies. A meaningful test also requires that the index profiles are at least tentatively detected: profiles that are consistent with being flat contain no information. In particular, we seek to match positive colour gradients (outskirts redder than the centre) to negative $\mathrm{H} \delta_{\mathrm{A}}$ gradients (outskirts older/more metal-rich than the centre). For simplicity, we refer to both positive colour gradients and negative $\mathrm{H} \delta_{\mathrm{A}}$ gradients as inverse gradients, and vice versa, negative colour gradients and positive $\mathrm{H} \delta_{\mathrm{A}}$ gradients are inside-out gradients.

Unfortunately, at the time of this writing, we can only present data for two galaxies (Fig. B1). The proposed approach is in fact limited by the unavailability of suitable data. As a precondition, we have reliable COSMOS F814W photometry for all the PSB galaxies in our sample, but, in general, suitable redder-wavelength images are unavailable. When available, these images have broader PSF than F814W photometry, and as a result, raw images show an artificially high fraction of galaxies with blue centers. Therefore we require also a reliable PSF deconvolution, which further limits the sample size. The overlap with the CANDELS Survey is insufficient (Grogin et al. 2011): out of seventeen PSB galaxies considered here, only galaxy 211263 M1 is present in CANDELS. This galaxy (not pictured) has flat $\mathrm{H} \delta_{\mathrm{A}}$ profile and negative colour gradient, and is therefore uninformative.

The COSMOS-DASH Survey (hereafter: DASH; Mowla et al. 2019), provides HST F160W imaging of the COSMOS field. Given that LEGA-C is selected from the COSMOS field, and that DASH currently covers $\approx 30 \%$ of this field, DASH data are available for five of our PSB galaxies. Of these, one is galaxy $211263 \mathrm{M} 1$, which we have already discarded. Of the remaining four galaxies, two more have flat $\mathrm{H} \delta_{\mathrm{A}}$ gradient (110805 M3 and $216730 \mathrm{M} 9$ ); moreover, both of these galaxies have unreliable GALFIT fits, in that the bestfit parameters reached the limits of the allowed range. The reason is probably that DASH is less deep than either COSMOS or CANDELS: the five $\sigma$ point-source detection limit for DASH is $H=25.2 \mathrm{mag}$ (Mowla et al. 2019), whereas CANDELS has $H=27 \mathrm{mag}$ (Grogin et al. 2011) and COSMOS has $I=27.2 \mathrm{mag}$ (Koekemoer et al. 2007). We are left with only two PSB galaxies. The first (258467 M13; Panel a) has both inside-out colour and inside-out $\mathrm{H} \delta_{\mathrm{A}}$ profile, whereas the second (250391 M11; Panel b), has both inverse colour and inverse $\mathrm{H} \delta_{\mathrm{A}}$ profile.

The agreement between the two reliable index gradients with the respective colour gradients is a reassuring consistency check for our methodology. We conclude that our detection of an inverse age gradient in the typical PSB is a robust result, and that space-based photometry may represent an alternative mean to investigate the inverse or inside-out structure of PSB galaxies. 


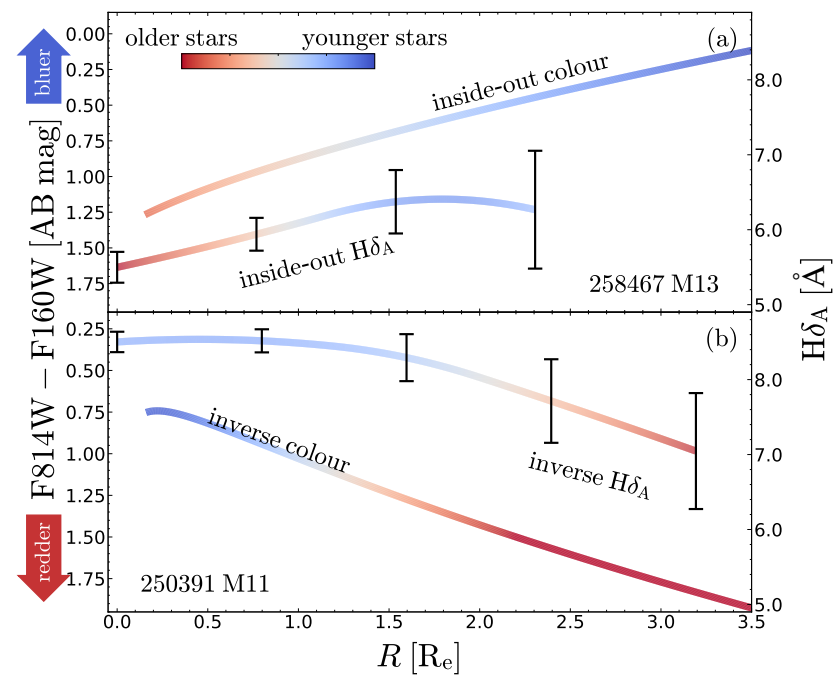

Figure B1. We show the clear connection between rest-frame optical colour profiles (lines with no error bars, left-hand scale) and $\mathrm{H} \delta_{\mathrm{A}}$ profiles (lines with error bars, right-hand scale), for the two PSB galaxies with both available colour profiles and significant $\mathrm{H} \delta_{\mathrm{A}}$ gradients. $258467 \mathrm{M} 13$ shows clear inside-out structure (outskirts bluer and with stronger $\mathrm{H} \delta_{\mathrm{A}}$ than the centre; Panel a), whereas 250391 M11 shows clear inverse structure (outskirts redder and with weaker $\mathrm{H} \delta_{\mathrm{A}}$ than the centre; Panel b). The lines are arbitrarily colour-coded so that bluer optical colour and stronger $\mathrm{H} \delta_{\mathrm{A}}$ absorption, corresponding to relatively younger stars, are rendered by bluer colour hues.

This paper has been typeset from a $\mathrm{T}_{\mathrm{E}} \mathrm{X} / \mathrm{LA} \mathrm{TEX}$ file prepared by the author. 